Supporting Information to Accompany:

\title{
Superior Reactivity of Thiosemicarbazides in the Synthesis of 2-Amino-1,3,4-Oxadiazoles:
}

Sarah J. Dolman, ${ }^{* \dagger}{ }^{\dagger}$ Francis Gosselin, ${ }^{\dagger}$ Paul D. O’Shea, ${ }^{\dagger}$ and Ian W. Davies ${ }^{\ddagger}$

(†) Department of Process Research, Merck Frosst Centre for Therapeutic Research

16711 route transcanadienne, Kirkland, Québec, Canada $\mathrm{H} 9 \mathrm{H} 3 \mathrm{L1}$

(¥) Department of Process Research, Merck Research Laboratories, P.O. Box 2000, Rahway, New Jersey, 07065

\section{Table of Contents}

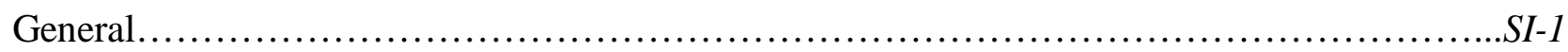

Representative procedure for hydrazide acylation with isocyanate or isothiocyanate............SI-2

Representative procedure for cyclization to oxadiazole....................................

Procedure for one-pot acylation / cyclization to oxadiazole...............................SI-2

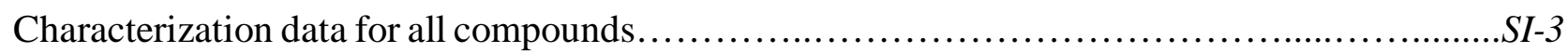

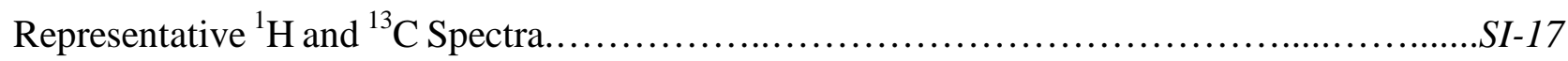

\section{General}

Reactions were carried out under an atmosphere of dry nitrogen. Reagents and solvents were used as received from commercial sources. ${ }^{1} \mathrm{H}$ NMR spectra were recorded on $400 \mathrm{MHz}$ spectrometer. Chemical shifts are reported in ppm from tetramethylsilane with the solvent resonance as the internal standard (DMSO- $d_{6}: \delta 2.49$ ). Data are reported as follows: chemical shift, multiplicity (s = singlet, $\mathrm{d}$ $=$ doublet, $\mathrm{t}=$ triplet, $\mathrm{q}=$ quartet, $\mathrm{br}=$ broad, $\mathrm{m}=$ multiplet $)$, coupling constants $(\mathrm{Hz})$ and integration.

${ }^{13} \mathrm{C}$ NMR spectra were recorded on a $100 \mathrm{MHz}$ spectrometer with complete proton decoupling. Chemical shifts are reported in ppm from tetramethylsilane with the solvent as the internal reference (DMSO- $d_{6}: \delta 39.5$ ). ${ }^{19} \mathrm{~F}$ NMR spectra were recorded on $375 \mathrm{MHz}$ spectrometer with complete proton decoupling. Chemical shifts are reported in ppm with $\alpha, \alpha, \alpha$-trifluorotoluene added as an internal reference $(\delta$-62.7). All compounds except were characterized using the same HPLC conditions: gradient elution: $\left(0.1 \% \mathrm{H}_{3} \mathrm{PO}_{4} / \mathrm{CH}_{3} \mathrm{CN}\right.$ 70:30 to 5:95 over $25 \mathrm{~min}$, hold $\left.5 \mathrm{~min}\right)$, flow rate $=2.0 \mathrm{~mL} / \mathrm{min}, \mathrm{T}=35^{\circ} \mathrm{C}, \mathrm{UV}$ detection at $210 \mathrm{~nm}$. 


\section{Representative Procedures:}

(1) Hydrazide acylation with isocyanate or isothiocyanate. Phenyl acetic acid hydrazide (2.0 g, $13.3 \mathrm{mmol})$ and benzyl isothiocyanate $(1.77 \mathrm{~mL}, 13.3 \mathrm{mmol})$ were combined in THF $(50 \mathrm{~mL})$ at room temperature. The resultant solution was stirred for $18 \mathrm{~h}$, then volatiles were removed in vacuo to afford an off-white solid. The solid was suspended and triturated in MTBE $(50 \mathrm{~mL})$ for $1 \mathrm{~h}$, then filtered to afford $3.80 \mathrm{~g}$ (95\% yield) of desired thiosemicarbazide.

(2) Cyclization to oxadiazole. N-Benzyl-phenyl acetic acid thiosemicarbazide 1 b (2.05 g, 6.85 mmol) tosyl chloride $(1.57 \mathrm{~g}, 8.22 \mathrm{mmol})$ and pyridine $(1.16 \mathrm{~mL}, 14.4 \mathrm{mmol})$ were combined in THF (30 mL) in a $100 \mathrm{~mL}$ round-bottom flask fitted with a magnetic stir-bar, reflux condenser and nitrogen inlet. The solution was heated in a $70{ }^{\circ} \mathrm{C}$ oil bath to bring the mixture to reflux for $20 \mathrm{~h}$, then cooled to room temperature. An aliquot of the crude reaction mixture indicated complete conversion (no thiosemicarbazide visible by LC) to the oxadiazole, with $97 \%$ assay yield. EtOAc $(20 \mathrm{~mL})$ and $1 \mathrm{~N} \mathrm{HCl}(20 \mathrm{~mL})$ were added; the mixture was vigorously stirred for $5 \mathrm{~min}$, then the aqueous layer was removed. The aqueous layer was back-extracted with EtOAc $(20 \mathrm{~mL})$, then the combined organic layers were flushed with heptane $(2 \times 75 \mathrm{~mL})$ and the material concentrated to an orange slurry. The material was dissolved in $\sim 10 \mathrm{~mL}$ THF and filtered over solka-floc. $\mathrm{HCl}$ (3.43 $\mathrm{mL}, 2.0 \mathrm{M}$ in diethyl ether) was added to the solution. After stirring $30 \mathrm{~min}$ at room temperature, a thin slurry was obtained. MTBE was added dropwise to the slurry $(25 \mathrm{~mL})$ and the resultant mixture stirred an additional $25 \mathrm{~min}$. The slurry was filtered to afford $1.74 \mathrm{~g}$ of shiny white powder (84\% yield).

(3) One-pot acylation / cyclization procedure. Phenyl acetic acid hydrazide (2.17 g, $14.4 \mathrm{mmol})$ and benzyl isothiocyanate $(1.92 \mathrm{~mL}, 14.4 \mathrm{mmol})$ were combined in THF $(50 \mathrm{~mL})$ in a $100-\mathrm{Ml}$ round-bottom flask at room temperature and stirred for $18 \mathrm{~h}$. Tosyl chloride $(3.30 \mathrm{~g}, 17.3 \mathrm{mmol})$ and pyridine $(2.45 \mathrm{~mL}, 30.3 \mathrm{mmol})$ were added to the reaction mixture. The flask was fitted with a reflux condenser and nitrogen inlet and submerged into a $70{ }^{\circ} \mathrm{C}$ oil bath. The solution was heated to reflux in an oil bath for $20 \mathrm{~h}$, then cooled to room temperature. An aliquot of the crude reaction mixture indicated complete conversion (no thiosemicarbazide visible by LC) to the oxadiazole, with $97 \%$ assay yield. EtOAc $(50 \mathrm{~mL})$ and $1 \mathrm{~N} \mathrm{HCl}(50 \mathrm{~mL})$ were added; the mixture was vigorously stirred for $5 \mathrm{~min}$, then the aqueous layer was removed. The aqueous layer was back-extracted with EtOAc (50 $\mathrm{mL})$, then the combined organic layers were flushed with heptane $(2 \times 150 \mathrm{~mL})$ and the material 
concentrated to an orange slurry. The material was dissolved in $\sim 35 \mathrm{~mL}$ THF and filtered over solkafloc. $\mathrm{HCl}(14.4 \mathrm{~mL}, 1.0 \mathrm{M}$ in diethyl ether) was added to the solution. After stirring $30 \mathrm{~min}$ at room temperature, a thin slurry was obtaned. MTBE was added dropwise to the slurry (70 $\mathrm{mL})$ and the resultant mixture stirred an additional $25 \mathrm{~min}$. The slurry was filtered to afford $3.70 \mathrm{~g}$ of shiny white powder (85\% yield).

\section{Characterization Data:}

$\boldsymbol{N}$-Benzyl-phenyl acetic acid semicarbazide (1a): ${ }^{1} \mathrm{H}$ NMR (400 MHz, dmso-d 6 ) $\delta 9.77$ (s, 1H)

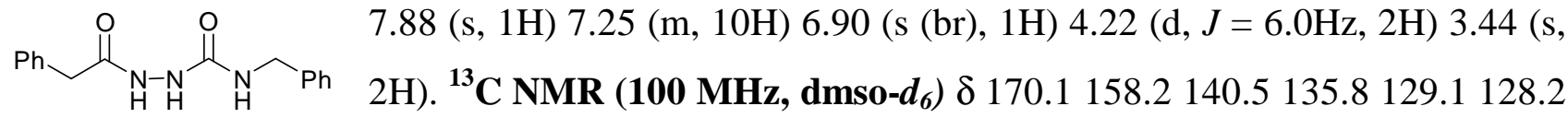
128.1126 .9126 .5126 .4 42.6. HPLC Retention time: 4.39 min. m.p. 197.0 - $198.0{ }^{\circ}$ C. IR 3333.9 (s) 3210.0 (s) 3030.2 (s) 1947.3 (w) 1695.9 (s) 1560.0 (s) 1452.4 (s) 1362.6 (m) 1274.0 (s) 1154.2 (m) $1074.6(\mathrm{~m})$. HRMS Calcd for $\mathrm{C}_{16} \mathrm{H}_{17} \mathrm{~N}_{3} \mathrm{O}_{2}$ : 284.1399. Found: 284.1401.

$\boldsymbol{N}$-Benzyl-phenyl acetic acid thio-semicarbazide (1b): ${ }^{\mathbf{1}} \mathrm{H}$ NMR (400 MHz, dmso- $\left.\boldsymbol{d}_{\boldsymbol{\sigma}}\right) \boldsymbol{\delta} 10.0$ (s,

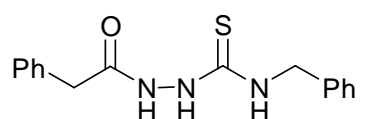
1H) $9.35(\mathrm{~s}, 1 \mathrm{H}) 8.51(\mathrm{~s}(\mathrm{br}), 1 \mathrm{H}) 7.25(\mathrm{~m}, 10 \mathrm{H}) 4.73(\mathrm{~d}, J=6.0 \mathrm{~Hz}, 2 \mathrm{H}) 3.47$ 128.0127 .0126 .5 46.7. HPLC Retention time: 6.78 min. m.p. $170.1-171.0{ }^{\circ} \mathrm{C}$. IR 3336.3 (s) 3201.9 (s) 3030.4 (s) 1948.5 (w) 1683.2 (s) 1653.5 (s) 1604.6 (m) 1555.2 (s) 1492.8 (s) 1378.1 (s) 1292.5 (s) 1186.8 (s) 1074.5 (s). HRMS Calcd for $\mathrm{C}_{16} \mathrm{H}_{17} \mathrm{~N}_{3} \mathrm{OS}$ : 300.1171. Found: 300.1174.

Benzyl-(5-benzyl-[1,3,4]oxadiazol-2-yl)-amine-HCl (2): ${ }^{1} \mathrm{H}$ NMR (400 MHz, dmso-d $\left.\boldsymbol{d}\right) \delta .99(\mathrm{t}$, $\overbrace{\mathrm{O}}^{\mathrm{N}} \overbrace{\mathrm{H}}^{\mathrm{N}} \widehat{\mathrm{C}}_{\mathrm{Ph}}$ $J=6.0 \mathrm{~Hz}, 1 \mathrm{H}) 7.30(\mathrm{~m}, 10 \mathrm{H}) 4.31(\mathrm{~d}, J=6.0 \mathrm{~Hz}, 2 \mathrm{H}) 4.04(\mathrm{~s}, 2 \mathrm{H}) .{ }^{13} \mathbf{C} \mathbf{N M R}$ 46.0 30.9 HPLC Retention time: 7.79 min. m.p. 157.0 - $157.5^{\circ} \mathrm{C}$. IR 2948.7 (m) 2550.5 (m) 1734.8 (s) 1714.7 (s) 1638.3 (s) 1496.8 (m) 1453.8 (m) 1415.1 (w) 1214.4 (w) 1011.0 (m). HRMS Calcd for $\mathrm{C}_{16} \mathrm{H}_{15} \mathrm{~N}_{3} \mathrm{O}: 266.1293$. Found: 266.1290.

$\boldsymbol{N}$-Cyclohexyl-phenyl acetic acid semicarbazide: ${ }^{1} \mathrm{H}$ NMR (400 MHz, dmso- $\left.\boldsymbol{d}_{\boldsymbol{\sigma}}\right) \boldsymbol{\delta} 9.72(\mathrm{~s}, 1 \mathrm{H})$<smiles>O=C(Cc1ccccc1)NNC(=O)NC1CCCCC1</smiles>
$7.64(\mathrm{~s}, 1 \mathrm{H}) 7.30(\mathrm{~m}, 5 \mathrm{H}) 6.02(\mathrm{~d}, J=8.0 \mathrm{~Hz}, 2 \mathrm{H}) 3.49(\mathrm{~s}, 2 \mathrm{H}) 3.35(\mathrm{~m}, 1 \mathrm{H})$ $1.71-1.49(\mathrm{~m}, 5 \mathrm{H}) 1.25-1.05(\mathrm{~m}, 5 \mathrm{H}) .{ }^{13} \mathrm{C}$ NMR (100 MHz, dmso-d $\left.\boldsymbol{d}_{\boldsymbol{\sigma}}\right) \delta$ 
169.7157 .1135 .9129 .0128 .2126 .447 .932 .9 25.2 24.5. HPLC Retention time: 4.84 min. m.p. 225.0 - $226.0{ }^{\circ} \mathrm{C}$. IR 3331.0 (s) 3216.1 (s) 3028.1 (m) 2928.6 (s) 2852.9 (s) 1692.1 (s) 1558.4 (s) 1496.6 (s) 1348.9 (m) 1272.1 (m) 1234.3 (s) 1166.4 (s) 1049.3 (m). HRMS Calcd for $\mathrm{C}_{15} \mathrm{H}_{21} \mathrm{~N}_{3} \mathrm{O}_{2}$ : 276.1712. Found: 276.1710 .

$\boldsymbol{N}$-Cyclohexyl-phenyl acetic acid thio-semicarbazide: ${ }^{1} \mathrm{H}$ NMR (400 MHz, dmso- $\left.\boldsymbol{d}_{\boldsymbol{\sigma}}\right) \boldsymbol{\delta} 9.91$ (s,<smiles>O=C(Cc1ccccc1)NNC(=S)NC1CCCCC1</smiles>
1H) $9.13(\mathrm{~s}, 1 \mathrm{H}) 7.24(\mathrm{~m}, 5 \mathrm{H}) 4.04(\mathrm{br}, 1 \mathrm{H}) 3.46(\mathrm{~s}, 2 \mathrm{H}) 1.79-1.57(\mathrm{~m}, 5 \mathrm{H})$ 1.33 - 1.11 (m, 5H). ${ }^{13}$ C NMR (100 MHz, dmso-d $) \delta 169.9135 .6129 .1$ 128.2 126.567 .052 .531 .8 25.1 24.7. HPLC Retention time: 7.42 min. m.p. $186.4-188.0{ }^{\circ}$ C. IR 3224.2 (s) 2933.8 (s) 2852.9 (s) 1681.9 (s) 1554.3 (s) 1498.2 (s) 1453.1 (m) 1408.1 (m) 1281.2 (m) 1151.8 (m) 1049.6 (m). HRMS Calcd for $\mathrm{C}_{15} \mathrm{H}_{21} \mathrm{~N}_{3} \mathrm{OS}$ : 292.1484. Found: 292.1479.

Cyclohexyl-(5-benzyl-[1,3,4] oxadiazol-2-yl)-amine-HCl (3): ${ }^{1} \mathrm{H}$ NMR (400 MHz, dmso-d $) \delta$ $\overbrace{}^{2-N}$ $7.29(\mathrm{~m}, 6 \mathrm{H}) 4.02(\mathrm{~s}, 2 \mathrm{H}) 3.26(\mathrm{~m}, 1 \mathrm{H}) 1.87(\mathrm{~m}, 2 \mathrm{H}) 1.67(\mathrm{~m}, 2 \mathrm{H}) 1.53(\mathrm{~m}, 2 \mathrm{H})$ $1.16(\mathrm{~m}, 5 \mathrm{H}) .{ }^{13} \mathrm{C}$ NMR (100 MHz, dmso-d $\left.\boldsymbol{d}_{6}\right) \delta 163.0127 .9135 .2128 .6127 .0$ 51.6 32.2 30.9 25.2 24.3. HPLC Retention time: 8.46 min. m.p. 199.0 - 202.0 ${ }^{\circ} \mathrm{C}$. IR 3233.4 (m) 3109.4 (m)2929.0 (s) 2857.4 (s) 2587.1 (s) 1717.4 (s) 1647.3 (s) 1497.2 (m) 1455.4 (m) 1337.5 (m) 1271.4 (m) 1081.8 (m) 1017.2 (s). HRMS Calcd for $\mathrm{C}_{15} \mathrm{H}_{19} \mathrm{~N}_{3} \mathrm{O}$ : 258.1606. Found: 258.1606.

N-tert-Butyl-phenyl acetic acid semicarbazide: ${ }^{1} \mathbf{H}$ NMR (400 MHz, dmso-d $) \delta 9.74(\mathrm{~s}, 1 \mathrm{H})$

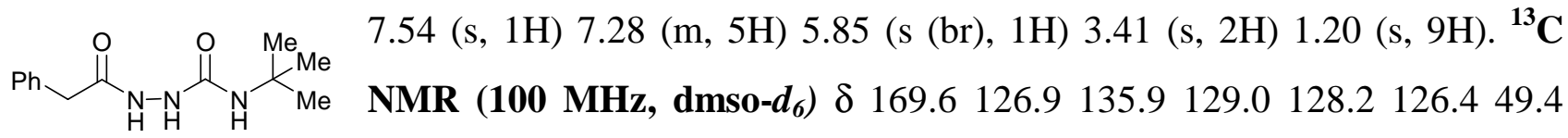
29.0. HPLC Retention time: 3.84 min. m.p. 190.1 - $191.2^{\circ}$ C. IR 3323.6 (s) 3248.5 (s) 3101.9 (m) 2971.5 (s) 2929.8 (w) 1694.0 (s) 1616.2 (s) 1575.6 (s) 1497.7 (m) 1391.7 (m) 1277.9 (s) 1223.2 (s) 1149.7 (s). HRMS Calcd for $\mathrm{C}_{13} \mathrm{H}_{19} \mathrm{~N}_{3} \mathrm{O}_{2}$ : 250.1556. Found: 250.1561 .

$N$-tert-Butyl-phenyl acetic acid thio-semicarbazide: ${ }^{1} \mathrm{H}$ NMR (400 MHz, dmso- $\left.d_{\sigma}\right) \delta 10.0(\mathrm{~s}, 1 \mathrm{H})$

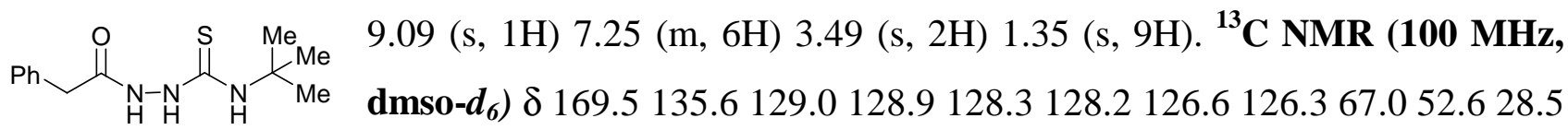
25.1. HPLC Retention time: 6.48 min. m.p. 137.3 - $138.4{ }^{\circ} \mathrm{C}$. IR 3223.7 (s) 2924.9 (s) 1673.7 (s) 
1551.8 (s) 1498.7 (s) 1460.4 (m) 1279.6 (m) 1149.2 (m) 1051.0 (m). RMS Calcd for $\mathrm{C}_{13} \mathrm{H}_{19} \mathrm{~N}_{3} \mathrm{OS}$ : 266.1327. Found: 266.1320.

$N$-tert-Butyl-(5-benzyl-[1,3,4]oxadiazol-2-yl)-amine-HCl (4): ${ }^{1} \mathrm{H}$ NMR (400 MHz, dmso- $\left.d_{6}\right) \delta$

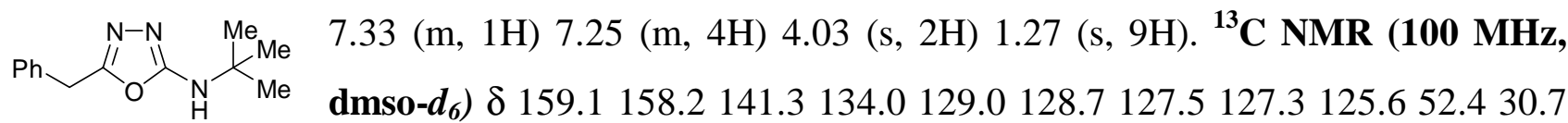
28.1. HPLC Retention time: 6.83 min. m.p. 138.1 - $140.2{ }^{\circ} \mathrm{C}$. IR 3237.0 (w) 3126.7 (s) 2985.1 (s) 2848.5 (s) 2642.9 (s) 1707.1 (s) 1684.9 (s) 1654.0 (w) 1638.7 (s) 1497.3 (m) 1376.3 (m) 1235.2 (m) 1082.2 (m). HRMS Calcd for $\mathrm{C}_{13} \mathrm{H}_{17} \mathrm{~N}_{3} \mathrm{O}: 232.1450$. Found: 232.1444.

$\boldsymbol{N}$-Benzyl-butyric acid semicarbazide: ${ }^{1} \mathrm{H}$ NMR (400 MHz, dmso- $\left.\boldsymbol{d}_{\boldsymbol{\sigma}}\right) \boldsymbol{\delta} 9.44(\mathrm{~s}, 1 \mathrm{H}) 7.78$ (s, $\left.1 \mathrm{H}\right)$

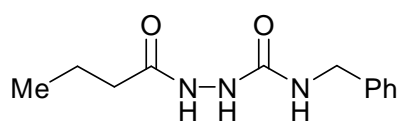
$7.25(\mathrm{~m}, 5 \mathrm{H}) 6.84(\mathrm{t}, J=6.0 \mathrm{~Hz}, 1 \mathrm{H}) 4.21(\mathrm{~d}, J=6.0 \mathrm{~Hz}, 2 \mathrm{H}) 2.07(\mathrm{t}, J=$ 7.4Hz, 2H) $1.52(\mathrm{~m}, 2 \mathrm{H}) 0.86(\mathrm{t}, J=7.4 \mathrm{~Hz}, 3 \mathrm{H}) .{ }^{13} \mathbf{C} \mathbf{N M R}(\mathbf{1 0 0} \mathbf{M H z}$, dmso-d $\left.\boldsymbol{d}_{\boldsymbol{6}}\right) \delta 172.1 \quad 158.3140 .6128 .1126 .9126 .542 .635 .118 .3$ 13.6. HPLC Retention time: 2.73 min. m.p. 205.1 - $208.4{ }^{\circ} \mathrm{C}$. IR 3390.5 (s) 3283.5 (m) 3192.0 (m) $2964.6(\mathrm{~m}) 2873.4(\mathrm{~m}) 1710.1$ (s) $1652.8(\mathrm{~s}) 1567.6(\mathrm{~s}) 1540.4(\mathrm{~s}) \mathbf{1 4 9 7 . 5}(\mathrm{m}) \mathbf{1 4 5 2 . 0}(\mathrm{m}) 1273.1(\mathrm{~m})$ 1236.1 (m) 1175.1 (w) 1052.6 (w). HRMS Calcd $\mathrm{C}_{12} \mathrm{H}_{17} \mathrm{~N}_{3} \mathrm{O}_{2}$ : 236.1399. Found: 236.1396.

$\boldsymbol{N}$-Benzyl-butyric acid thio-semicarbazide: ${ }^{\mathbf{1}} \mathbf{H}$ NMR (400 MHz, dmso- $\left.\boldsymbol{d}_{\boldsymbol{6}}\right) \boldsymbol{\delta} 9.67$ (s, $\left.1 \mathrm{H}\right) 9.25$ (s, $1 \mathrm{H}) 8.37(\mathrm{~s}(\mathrm{br}), 1 \mathrm{H}) 7.27(\mathrm{~m}, 5 \mathrm{H}) 4.70(\mathrm{~d}, J=6.0 \mathrm{~Hz}, 2 \mathrm{H}) 2.10(\mathrm{t}, J=$
$\mathrm{Me}$ dmso- $_{\boldsymbol{\sigma}}$ ) $\delta 172.0139 .4128 .0127 .0126 .646 .635 .2$ 18.0 13.7. HPLC Retention time: $4.55 \mathrm{~min}$. m.p. 160.8 - $161.9^{\circ} \mathrm{C}$. IR 3504.3 (s) 3157.9 (s) 2960.1 (s) 1668.6 (s) 1568.2 (s) 1451.8 (s) 1392.2 (m) 1350.6 (s) 1279.2 (s) 1236.3 (s) 1189.3 (s) 1063.5 (m). HRMS Calcd $\mathrm{C}_{12} \mathrm{H}_{17} \mathrm{~N}_{3} \mathrm{OS}$ : 252.1171. Found: 252.1175.

$N$-Benzyl-(5-butyl-[1,3,4]oxadiazol-2-yl)-amine (5): ${ }^{1} \mathrm{H}$ NMR (400 MHz, dmso- $\left.d_{6}\right) \delta 7.92(\mathrm{t}, J=$ $\overbrace{\mathrm{O}}^{\mathrm{N}-\mathrm{N}} \overbrace{\mathrm{H}}^{\mathrm{N}} \overbrace{\mathrm{Ph}}$ $4.8 \mathrm{~Hz}, 1 \mathrm{H}) 7.31(\mathrm{~m}, 4 \mathrm{H}) 7.25(\mathrm{~m}, 1 \mathrm{H}) 4.33(\mathrm{~d}, J=4.8 \mathrm{~Hz}, 2 \mathrm{H}) 2.60(\mathrm{t}, J=$ $5.6 \mathrm{~Hz}, 2 \mathrm{H}) 1.60(\mathrm{~m}, 2 \mathrm{H}) 0.90(\mathrm{t}, J=6.0 \mathrm{~Hz}, 3 \mathrm{H}) .{ }^{13} \mathrm{C}$ NMR (100 MHz, 
dmso-d $\left._{\boldsymbol{\sigma}}\right) \boldsymbol{\delta} 163.4159 .6138 .9128 .3127 .3127 .046 .026 .4$ 19.4 13.3. HPLC Retention time: 5.67 min. m.p. $83.4-84.8^{\circ} \mathrm{C}$. IR 3214.8 (m) 3033.5 (m) 2963.8 (m) 1635.4 (s) 1584.5 (s) 1455.6 (m) 1333.7 (w) 1191.2 (m). HRMS Calcd $\mathrm{C}_{12} \mathrm{H}_{15} \mathrm{~N}_{3} \mathrm{O}$ : 218.1293. Found: 218.1295.

$N$-Benzyl-2-hydroxy-2-methyl-butyric acid semicarbazide: ${ }^{1} \mathrm{H}$ NMR (400 MHz, dmso- $\left.d_{6}\right) \delta$

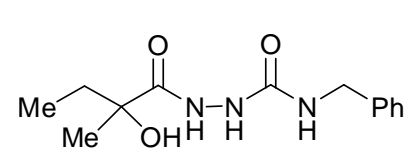

$9.27(\mathrm{~s}, 1 \mathrm{H}) 7.80(\mathrm{~s}, 1 \mathrm{H}) 7.24(\mathrm{~m}, 5 \mathrm{H}) 6.64(\mathrm{t}, J=6.0 \mathrm{~Hz}, 1 \mathrm{H}) 5.09(\mathrm{~s}, 1 \mathrm{H})$ $4.23(\mathrm{~d}, J=6.0 \mathrm{~Hz}, 2 \mathrm{H}) 1.66(\mathrm{~m}, 1 \mathrm{H}) 1.46(\mathrm{~m}, 1 \mathrm{H}) 1.23(\mathrm{~s}, 3 \mathrm{H}) 0.80(\mathrm{t}, J=$ 7.2Hz, 3H). ${ }^{13} \mathbf{C}$ NMR (100 MHz, dmso-d $)_{6} \delta 175.4158 .5140 .4128 .1$ 126.9126 .674 .642 .632 .925 .9 7.9. HPLC Retention time: 2.33 min. m.p. 144.5 - $145.2{ }^{\circ}$ C. IR 3368.7 (s) 3976.4 (w) 1682.7 (s) 1651.6 (s) 1504.8 (m) 1451.4 (m) 1364.6 (w) 1180.6 (m) 1044.1 (w). HRMS Calcd $\mathrm{C}_{13} \mathrm{H}_{19} \mathrm{~N}_{3} \mathrm{O}_{3}: 266.1505$. Found: 266.1501 .

$N$-Benzyl-2-hydroxy-2-methyl-butyric acid thio-semicarbazide: ${ }^{1} \mathrm{H}$ NMR (400 MHz, dmso- $\left.d_{6}\right)$

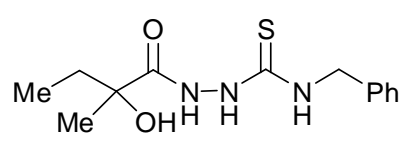
$\delta 9.76(\mathrm{~s}, 1 \mathrm{H}) 9.37$ (br s, 1H) $7.95(\mathrm{~s}, 1 \mathrm{H}) 7.26(\mathrm{~m}, 5 \mathrm{H}) 5.13(\mathrm{~s}, 1 \mathrm{H}) 4.73$ MHz, dmso- $\left.\boldsymbol{d}_{\boldsymbol{\sigma}}\right) \boldsymbol{\delta} 182.0139 .0128 .1126 .9126 .7$ 74.8 46.7 32.7 25.5 7.9. HPLC Retention time: 3.85 min. m.p. $132.5-132.7^{\circ} \mathrm{C}$. IR 3519.1 (w) 3261.8 (s) 2975.0 (m) 1679.4 (s) 1550.3 (s) 1495.2 (m) 1306.8 (w) 1133.0 (m). HRMS Calcd $\mathrm{C}_{13} \mathrm{H}_{19} \mathrm{~N}_{3} \mathrm{O}_{2} \mathrm{~S}$ : 282.1276. Found: 282.1270.

N-Benzyl-(5-(2-hydroxy-2-methyl)-butyl-[1,3,4]oxadiazol-2-yl)-amine·HCl (6): ${ }^{1} \mathrm{H}$ NMR (400 $\overbrace{\mathrm{Me}}^{\mathrm{N}-\mathrm{O}}$ MHz, dmso-d $\left.\boldsymbol{d}_{\boldsymbol{\sigma}}\right) \delta 9.65(\mathrm{br} \mathrm{s}, 1 \mathrm{H}) 7.40(\mathrm{~d}, J=7.6 \mathrm{~Hz}, 2 \mathrm{H}) 7.35(\mathrm{t}, J=7.6 \mathrm{~Hz}$, $0.81(\mathrm{t}, J=7.6 \mathrm{~Hz}, 3 \mathrm{H}) .{ }^{13} \mathbf{C}$ NMR (100 MHz, dmso-d $\left.\boldsymbol{d}_{\boldsymbol{6}}\right) \delta 163.3160 .9137 .4128 .5127 .7127 .569 .4$ 46.233 .024 .7 8.1. HPLC Retention time: 3.43 min. m.p. 132.0 - $133.1^{\circ}$ C. IR 3163.0 (s) 2971.4 (s) 1722.3 (s) 1623.9 (w) 1455.6 (m) 1381.9 (m) 1133.9 (s) 1014.1 (s). HRMS Calcd $\mathrm{C}_{13} \mathrm{H}_{17} \mathrm{~N}_{3} \mathrm{O}_{2}$ : 248.1399. Found: 248.1394.

$N$-Benzyl-tert-butyric acid semicarbazide: ${ }^{1} \mathrm{H}$ NMR (400 MHz, dmso-d $\left.\boldsymbol{d}_{6}\right) \boldsymbol{\delta} 9.23$ (s, $\left.1 \mathrm{H}\right) 7.65$ (s,

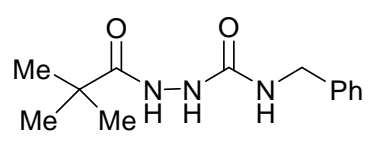
1H) $7.25(\mathrm{~m}, 5 \mathrm{H}) 6.66(\mathrm{t}, J=6.1 \mathrm{~Hz}, 1 \mathrm{H}) 4.23(\mathrm{~d}, J=6.1 \mathrm{~Hz}, 2 \mathrm{H}) 1.12(\mathrm{~s}$, 27.1. HPLC Retention time: 3.52 min. m.p. 153.2 - $154.5^{\circ} \mathrm{C}$. IR 3290.7 (s) 2965.7 (m) 1706.7 (s) 
1625.5 (s) 1545.5 (s) 1452.7 (m) 1369.8 (m) 1235.0 (m). HRMS Calcd $\mathrm{C}_{13} \mathrm{H}_{19} \mathrm{~N}_{3} \mathrm{O}_{2}$ : 250.1556. Found: 250.1552 .

$\boldsymbol{N}$-Benzyl-tert-butyric acid thio-semicarbazide: ${ }^{1} \mathrm{H}$ NMR (400 MHz, dmso-d $\left.\boldsymbol{d}_{6}\right) \boldsymbol{\delta} 9.49$ (s, $\left.1 \mathrm{H}\right)$

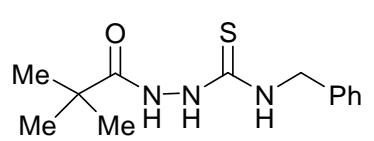
$9.20(\mathrm{~d}, J=10.7 \mathrm{~Hz}, 1 \mathrm{H}) 8.04(\mathrm{~s}, 1 \mathrm{H}) 7.35(\mathrm{~d}, J=54.4 \mathrm{~Hz}, 4 \mathrm{H}) 7.22(\mathrm{~d}, J=$ $6.5 \mathrm{~Hz}, 1 \mathrm{H}) 4.75(\mathrm{~d}, J=5.9 \mathrm{~Hz}, 2 \mathrm{H}) 1.15(\mathrm{~s}, 9 \mathrm{H}) .{ }^{13} \mathrm{C}$ NMR (100 MHz, dmso- $\left._{\boldsymbol{\sigma}}\right) \delta 139.4,128.2,128.0,127.2,126.9,126.6,46.7,37.7,27.0$. HPLC

Retention time: 5.86 min. m.p. 142.5 - $143.8{ }^{\circ} \mathrm{C}$. IR 3289.2 (s) 2966.5 (s) 1635.8 (s) 1540.1 (s) 1457.3 (s) 1274.6 (s) 1078.8 (s). HRMS Calcd $\mathrm{C}_{13} \mathrm{H}_{19} \mathrm{~N}_{3} \mathrm{OS}$ : 266.1327. Found: 266.1321.

$N$-Benzyl-(5-tert-butyl-[1,3,4] oxadiazol-2-yl)-amine·HCl (7): ${ }^{1} \mathrm{H}$ NMR (400 MHz, dmso- $\left.d_{6}\right) \delta$

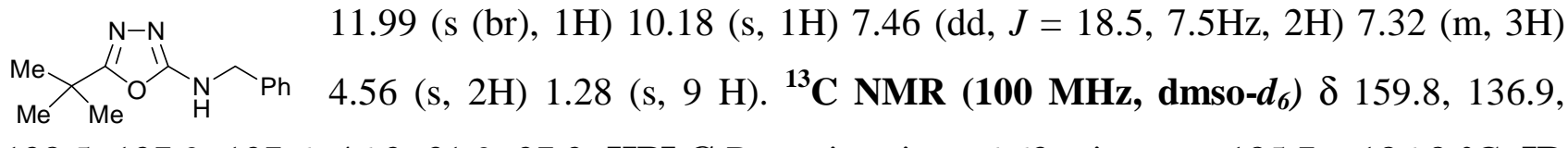
128.5, 127.9, 127.6, 46.2, 31.9, 27.2. HPLC Retention time: 6.63 min. m.p. 185.7 - $186.8^{\circ}$ C. IR 2970.9 (s) 1718.6 (s) 1629.9 (s) 1456.2 (s) 1367.9 (s) 1238.4 (m) 1145.2 (s). HRMS Calcd $\mathrm{C}_{13} \mathrm{H}_{17} \mathrm{~N}_{3} \mathrm{O}: 232.1450$. Found: 232.1447.

$\boldsymbol{N}$-Phenyl-phenyl acetic acid semicarbazide: ${ }^{\mathbf{H}} \mathbf{H}$ NMR (400 MHz, dmso-d $\left.\boldsymbol{d}_{\boldsymbol{\sigma}}\right) \boldsymbol{\delta} 9.95$ (s, $\left.1 \mathrm{H}\right) 8.72$ (s,<smiles>O=C(Cc1ccccc1)NNC(=O)Nc1ccccc1</smiles>
1H) $8.08(\mathrm{~s}, 1 \mathrm{H}) 7.43(\mathrm{~d}, J=8.0 \mathrm{~Hz}, 2 \mathrm{H}) 7.31(\mathrm{~m}, 5 \mathrm{H}) 7.24(\mathrm{t}, J=7.6 \mathrm{~Hz}$, 2H) $6.94(\mathrm{t}, J=7.6 \mathrm{~Hz}, 1 \mathrm{H}) 3.49(\mathrm{~s}, 2 \mathrm{H}) .{ }^{13} \mathrm{C}$ NMR (100 MHz, dmso-d $) \delta$ 170.1155 .3139 .6135 .7129 .1128 .7128 .2126 .5 121.9 118.4. HPLC Retention time: $4.34 \mathrm{~min}$. m.p. 210.0 - $211.3{ }^{\circ} \mathrm{C}$. IR 3276.2 (s) 3037.6 (s) 1651.3 (s) 1598.0 (s) 1562.0 (s) 1495.8 (s) 1453.9 (m) 1312.7 (m) 1236.5 (m) 1117.1 (m). HRMS Calcd $\mathrm{C}_{15} \mathrm{H}_{15} \mathrm{~N}_{3} \mathrm{O}_{2}$ : 270.1243. Found: 270.1244.

$N$-Phenyl-phenyl acetic acid thio-semicarbazide: ${ }^{1} \mathrm{H}$ NMR (400 MHz, dmso-d $\left.\boldsymbol{d}_{\boldsymbol{\sigma}}\right) \boldsymbol{\delta} 10.16(\mathrm{~s}, 1 \mathrm{H})$<smiles>O=C(Cc1ccccc1)NNC(=S)Nc1ccccc1</smiles>
$9.66(\mathrm{~s}(\mathrm{br}), 1 \mathrm{H}) 9.60(\mathrm{~s}, 1 \mathrm{H}) 7.43(\mathrm{~d}, J=7.6 \mathrm{~Hz}, 2 \mathrm{H}) 7.31(\mathrm{~m}, 6 \mathrm{H}) 7.24(\mathrm{~m}$, 1H) $7.16(\mathrm{t}, J=7.2 \mathrm{~Hz}, 1 \mathrm{H}) 3.52(\mathrm{~s}, 2 \mathrm{H}) .{ }^{13} \mathrm{C}$ NMR (100 MHz, dmso-d $) \delta$ 170.1139 .1135 .5129 .3128 .2 126.5. HPLC Retention time: 5.53 min. m.p. $160.4-162.2^{\circ} \mathrm{C}$. IR 3302.2 (s) 3224.0 (s) 3059.8 (w) 1700.5 (m) 1663.1 (s) 1627.9 (m) 1540.2(s) 1499.0 (s) 1361.5 (s) 1226.9 (w) 1161.2 (w). HRMS Calcd $\mathrm{C}_{15} \mathrm{H}_{15} \mathrm{~N}_{3} \mathrm{OS}$ : 286.1014. Found: 286.1010. 
$\boldsymbol{N}$-Phenyl-(5-benzyl-[1,3,4]oxadiazol-2-yl)-amine-HCl (8): ${ }^{1} \mathrm{H}$ NMR (400 MHz, dmso-d 6 ) $\delta 10.37$ $\mathrm{CH}_{\mathrm{O}}^{\mathrm{N}} \mathrm{C}_{\mathrm{H}}^{\mathrm{N}}$ $(\mathrm{s}, 1 \mathrm{H}) 7.51(\mathrm{~d}, J=8.0 \mathrm{~Hz}, 2 \mathrm{H}) 7.33(\mathrm{~m}, 7 \mathrm{H}) 6.96(\mathrm{t}, J=7.4 \mathrm{~Hz}, 1 \mathrm{H}) 4.16(\mathrm{~s}$, 128.7127 .1121 .6116 .8 30.7. HPLC Retention time: 8.19 min. m.p. 178.1 - $180.6{ }^{\circ}$ C. IR 3029.6 (m) 2928.6 (s) 2396.1 (m) 1836.9 (m) 1672.0 (s) 1621.7 (s) 1595.0 (s) 1500.9 (s) 1455.6 (s) 1246.9 (w) 1106.1 (w) 1015.4 (s). HRMS Calcd $\mathrm{C}_{15} \mathrm{H}_{13} \mathrm{~N}_{3} \mathrm{O}$ : 252.1137. Found: 252.1130.

$\boldsymbol{N}$-Phenyl acetic acid semicarbazide: ${ }^{1} \mathrm{H}$ NMR (400 MHz, dmso- $\left.\boldsymbol{d}_{\boldsymbol{6}}\right) \boldsymbol{\delta} 9.62(\mathrm{~s}, 1 \mathrm{H}) 8.69(\mathrm{~s}, 1 \mathrm{H})$<smiles>CC(=O)NNC(=O)Nc1ccccc1</smiles>
$7.95(\mathrm{~s}, 1 \mathrm{H}) 7.42(\mathrm{~d}, J=7.6 \mathrm{~Hz}, 2 \mathrm{H}) 7.24(\mathrm{t}, J=8.0 \mathrm{~Hz}, 2 \mathrm{H}) 6.94(\mathrm{t}, J=7.6 \mathrm{~Hz}$, 1H) $1.86(\mathrm{~s}, 3 \mathrm{H}) .{ }^{\mathbf{1 3}} \mathrm{C}$ NMR (100 MHz, dmso-d $\left.\boldsymbol{d}_{6}\right) \delta 169.3155 .4139 .6128 .6$ 121.9 118.5 20.6. HPLC Retention time: 1.75 min. m.p. 170.2 - $171.4{ }^{\circ}$ C. IR 3297.0 (s) 3041.7 (s) 1653.7 (s) 1560.2 (s) 1370.5 (s) 1234.2 (s) 1177.1 (m). HRMS Calcd $\mathrm{C}_{9} \mathrm{H}_{11} \mathrm{~N}_{3} \mathrm{O}_{2}$ : 194.0930. Found: 194.0929.

$\boldsymbol{N}$-Phenyl acetic acid thio-semicarbazide: ${ }^{1} \mathrm{H}$ NMR (400 MHz, dmso-d $\boldsymbol{d}_{\boldsymbol{\sigma}} \boldsymbol{\delta} 9.86$ (s, $\left.1 \mathrm{H}\right) 9.60$ (s $(\mathrm{br}), 1 \mathrm{H}) 9.51(\mathrm{~s}, 1 \mathrm{H}) 7.42(\mathrm{~d}, J=8.0 \mathrm{~Hz}, 2 \mathrm{H}) 7.32(\mathrm{t}, J=8.0 \mathrm{~Hz}, 2 \mathrm{H}) 7.15(\mathrm{t}, J$
$=7.2 \mathrm{~Hz}, 1 \mathrm{H}) 1.88(\mathrm{~s}, 3 \mathrm{H}) .{ }^{\mathbf{1 3}} \mathbf{C} \mathbf{N M R}\left(\mathbf{1 0 0} \mathbf{M H z}, \mathbf{d m s o}-\boldsymbol{d}_{\boldsymbol{\sigma}}\right) \delta 181.0169 .2$ 139.2128 .1125 .8 125.1 21.4. HPLC Retention time: 2.06 min. m.p. 175.9 - $176.7{ }^{\circ}$ C. IR 3260.4 (s) 3031.5 (s) 1700.7 (s) 1589.9 (m) 1496.7 (s) 1366.2 (m) 1248.2 (s) 1121.0 (m) 1072.0 (m). HRMS Calcd $\mathrm{C}_{9} \mathrm{H}_{11} \mathrm{~N}_{3} \mathrm{OS}: 210.0701$. Found: 210.0699.

$N$-Phenyl-(5-methyl-[1,3,4]oxadiazol-2-yl)-amine-HCl (9): ${ }^{1} \mathrm{H}$ NMR (400 MHz, dmso-d $d_{6} \delta$ $\overbrace{\mathrm{O}}^{\mathrm{N}} \mathrm{N}_{\mathrm{H}}^{\mathrm{N}}$ $10.51($ br s, 1H) $7.54(\mathrm{~d}, J=8.0 \mathrm{~Hz}, 2 \mathrm{H}) 7.30(\mathrm{t}, J=7.6 \mathrm{~Hz}, 2 \mathrm{H}) 6.96(\mathrm{t}, J=$ 7.2Hz, 1H) 2.37 (s, 3H). ${ }^{\mathbf{1 3}} \mathbf{C}$ NMR (100 MHz, dmso-d $\left.)\right) \delta 159.7157 .0138 .8$ 129.1121 .8117 .1 10.5. HPLC Retention time: 3.44 min. m.p. 160.0 - $162.9{ }^{\circ} \mathrm{C}$. IR 3183.9 (s) 3048.7 (s) 1796.8 (m) 1675.8 (s) 1559.8 (s) 1387.3 (m) 1226.1 (m) 1012.4 (m). HRMS Calcd $\mathrm{C}_{9} \mathrm{H}_{9} \mathrm{~N}_{3} \mathrm{O}$ : 176.0824 . Found: 176.0826 .

$N$-Phenyl-trifluoromethyl acetic acid semicarbazide: ${ }^{1} \mathrm{H}$ NMR (400 MHz, dmso- $\left.d_{6}\right) \delta 8.76(\mathrm{~s}$, 1H) $7.95(\mathrm{~s}, 1 \mathrm{H}) 7.47(\mathrm{~d}, J=8.0 \mathrm{~Hz}, 2 \mathrm{H}) 7.24(\mathrm{t}, J=8.0 \mathrm{~Hz}, 2 \mathrm{H}) 6.95(\mathrm{t}, J=7.6 \mathrm{~Hz}, 1 \mathrm{H}) .{ }^{13} \mathbf{C} \mathbf{N M R}$

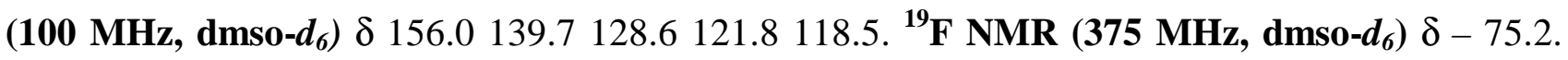




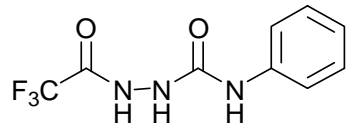
(m).
HPLC Retention time: 4.40 min. m.p. 265.8 - $266.6{ }^{\circ} \mathrm{C}$. IR 3298.5 (s) 3093.9 (m) 1675.0 (s) 1558.4 (s) 1446.6 (s) 1337.9 (m) 1282.0 (m) 1173.9

$\boldsymbol{N}$-Phenyl- trifluoromethyl acetic acid thio-semicarbazide: ${ }^{1} \mathrm{H}$ NMR (400 MHz, dmso- $\left.\boldsymbol{d}_{6}\right) \boldsymbol{\delta} 9.87$<smiles>O=C(NNC(=S)Nc1ccccc1)C(F)(F)F</smiles>
(s (br), 1H) 9.67 (s (br), 1H) $7.53(\mathrm{~d}, J=7.6 \mathrm{~Hz}, 2 \mathrm{H}) 7.33(\mathrm{t}, J=8.0 \mathrm{~Hz}, 2 \mathrm{H})$ $7.14(\mathrm{t}, J=7.6 \mathrm{~Hz}, 1 \mathrm{H}) .{ }^{13} \mathbf{C}$ NMR (100 MHz, dmso-d $\left.\boldsymbol{d}_{\boldsymbol{6}}\right) \delta 170.1155 .3139 .2$ 128.2 124.9. ${ }^{19}$ F NMR (375 MHz, dmso-d $\left.\boldsymbol{d}_{6}\right) \delta$ - 75.3. HPLC Retention time: 6.60 min. m.p. 196.7 - $196.9{ }^{\circ} \mathrm{C} . \mathbf{I R} 3212.1$ (s) 2009.6 (m) 1597.6 (m) 1546.9 (s) 1508.9 (s) 1451.9 (m) 1336.4 (m) $1243.9(\mathrm{~m}) 1189.3(\mathrm{~s})$.

N-Phenyl-(5- trifluoromethyl-[1,3,4]oxadiazol-2-yl)-amine·HCl (10): ${ }^{1} \mathrm{H}$ NMR (400 MHz, ${ }^{3} \mathrm{C}$ dmso-d $\boldsymbol{d}_{\boldsymbol{\sigma}} \delta 7.52(\mathrm{~d}, J=7.6 \mathrm{~Hz}, 2 \mathrm{H}) 7.34(\mathrm{t}, J=7.6 \mathrm{~Hz}, 2 \mathrm{H}) 7.03(\mathrm{t}, J=7.6 \mathrm{~Hz}$, 1H). ${ }^{13}$ C NMR (100 MHz, dmso-d $)$ ) $\delta 156.4140 .1129 .3128 .2125 .5122 .6$ 118.2. ${ }^{19}$ F NMR (375 MHz, dmso-d $\left.\boldsymbol{d}_{6}\right) \delta$ - 62.7. HPLC Retention time: 7.57 min. m.p. $125.7-$ $128.5^{\circ} \mathrm{C} . \mathbf{I R} 3392.9$ (m) 3183.0 (s) 2920.7 (s) 1603.9 (s) 1560.2 (s) 1496.9 (s) 1370.8 (m) 1260.0 (m) $1189.8(\mathrm{~s}) 1123.2(\mathrm{~m}) 1035.1(\mathrm{~m})$.

$\boldsymbol{N}$-Benzyl-4-fluoro-benzoic acid semicarbazide: ${ }^{1} \mathrm{H}$ NMR (400 MHz, dmso-d $)_{6} \delta 10.21(\mathrm{~s}, 1 \mathrm{H})$ $\overbrace{H}$ $7.97(\mathrm{~m}, 3 \mathrm{H}) 7.26(\mathrm{~m}, 8 \mathrm{H}) 4.24(\mathrm{~d}, J=6.0 \mathrm{~Hz}, 2 \mathrm{H}) .{ }^{13} \mathrm{C}$ NMR (100 MHz, dmso-d $\left._{\boldsymbol{\sigma}}\right) \delta 165.4162 .9158 .5140 .6130 .3(\mathrm{~d}, J=8.9 \mathrm{~Hz}) 129.3128 .1$ $126.9126 .5115 .3(\mathrm{~d}, J=21.6 \mathrm{~Hz}) 42.6 .{ }^{19} \mathbf{F}$ NMR (375 MHz, dmso-d $\left.)\right) \delta$ - 110.2. HPLC Retention time: 4.43 min. m.p. 229.5 - $230.4{ }^{\circ} \mathrm{C}$. IR 3304.6 (s) 3066.5 (m) 3031.3 (m) 2921.2 (w) 1652.7 (s) 1604.1 (s) 1501.0 (s) 1455.5 (m) 1340.4 (m) 1233.4 (s) 1162.5 (s). HRMS Calcd $\mathrm{C}_{15} \mathrm{H}_{14} \mathrm{FN}_{3} \mathrm{O}_{2}$ : 288.1148. Found: 288.1147.

$\boldsymbol{N}$-Benzyl-4-fluoro-benzoic acid thio-semicarbazide: ${ }^{\mathbf{1}} \mathrm{H}$ NMR (400 MHz, dmso-d $\boldsymbol{d}_{\boldsymbol{\sigma}} \boldsymbol{\delta} 10.44$ (s,<smiles>O=C(NNC(=S)Nc1ccccc1)c1ccc(F)cc1</smiles>
1H) $9.45(\mathrm{~s}, 1 \mathrm{H}) 8.67(\mathrm{~s}, 1 \mathrm{H}) 7.98(\mathrm{~m}, 2 \mathrm{H}) 7.33(\mathrm{~m}, 7 \mathrm{H}) 4.73(\mathrm{~d}, J=$ 6.0Hz, 2H). ${ }^{13} \mathrm{C}$ NMR (100 MHz, dmso-d $\left.)\right) \delta 165.0139 .4130 .6130 .5$ $128.0127 .0126 .6115 .3115 .146 .7 .{ }^{19}$ F NMR (375 MHz, dmso- $\left.d_{6}\right) \delta-$ 
109.9. HPLC Retention time: 6.79 min. m.p. 171.9 - $173.9^{\circ} \mathrm{C}$. IR 3376.5 (m) 3219.7 (s) 2977.7 (m) 1680.8 (s) 1603.6 (s) 1540.9 (s) 1484.9 (s) 1454.0 (m) 1265.4 (m) 1164.6 (m) 1104.5 (w). HRMS Calcd $\mathrm{C}_{15} \mathrm{H}_{14} \mathrm{FN}_{3} \mathrm{OS}: 304.0920$. Found: 304.0918.

$N$-Benzyl-(5-(4-fluorophenyl)-[1,3,4]oxadiazol-2-yl)-amine (11): ${ }^{1} \mathrm{H}$ NMR (400 MHz, dmso- $\left.d_{6}\right) \delta$ (1) $8.36(\mathrm{t}, J=6.4 \mathrm{~Hz}, 1 \mathrm{H}) 7.84(\mathrm{dd}, J=5.2 \mathrm{~Hz}, J=8.8 \mathrm{~Hz}, 2 \mathrm{H}) 7.35(\mathrm{~m}, 5 \mathrm{H})$ $7.26(\mathrm{t}, J=7.0 \mathrm{~Hz}, 1 \mathrm{H}) 4.44(\mathrm{~d}, J=6.4 \mathrm{~Hz}, 2 \mathrm{H}) .{ }^{13} \mathrm{C}$ NMR $(\mathbf{1 0 0} \mathbf{M H z}, \mathbf{d m s o}-$ $\left.\boldsymbol{d}_{\boldsymbol{\sigma}}\right) \delta 163.6138 .7128 .4127 .7127 .6127 .4127 .2120 .9166 .6116 .346 .0 .{ }^{19} \mathbf{F}$ NMR (375 MHz, dmso- $\left.d_{6}\right) \delta-111.2$. HPLC Retention time: 8.65 min. m.p. $128.5-130.6{ }^{\circ} \mathrm{C}$. IR 3232.1 (m) 3030.7 (m) 1715.2 (s) 1628.0 (s) 1502.8 (s) 1364.5 (w) 1231.1 (s) 1145.8 (m) 1033.2 (m). HRMS Calcd $\mathrm{C}_{15} \mathrm{H}_{12} \mathrm{FN}_{3} \mathrm{O}: 270.1043$. Found: 270.1041.

$\boldsymbol{N}$-Benzyl-2-methyl-benzoic acid semicarbazide: ${ }^{1} \mathrm{H}$ NMR (400 MHz, dmso- $\left.\boldsymbol{d}_{6}\right) \boldsymbol{\delta} 9.82(\mathrm{~s}, 1 \mathrm{H})$ Me $8.00(\mathrm{~s}, 1 \mathrm{H}) 7.46(\mathrm{~d}, J=7.2 \mathrm{~Hz}, 1 \mathrm{H}) 7.30(\mathrm{~m}, 8 \mathrm{H}) 6.93(\mathrm{br}, 1 \mathrm{H}) 4.27$ (d, $J=$ 6.0Hz, 2H) 2.37 (s, 3H). ${ }^{13} \mathbf{C}$ NMR (100 MHz, dmso-d $\left.\boldsymbol{d}_{\boldsymbol{\sigma}}\right) \delta 169.0158 .3$ 140.6136 .1130 .5129 .8128 .1127 .6126 .9126 .5125 .342 .7 19.4. HPLC Retention time: 4.60 min. m.p. 186.9 - $188.2{ }^{\circ} \mathrm{C}$. IR 3369.5 (s) 3309.5 (s) 3214.1 (s) 3009.5 (s) 2935.5 (m) 1707.2 (s) 1635.2 (s) 1540.3 (s) 1428.7 (s) 1361.2 (w) 1265.8 (s) $1238.2(\mathrm{~s}) 1131.5(\mathrm{~m})$ 1052.4 (m). HRMS Calcd $\mathrm{C}_{16} \mathrm{H}_{17} \mathrm{~N}_{3} \mathrm{O}_{2}: 284.1399$. Found: 284.1394 .

$\boldsymbol{N}$-Benzyl-2-methyl-benzoic acid thio-semicarbazide: ${ }^{1} \mathrm{H}$ NMR $\left(400 \mathrm{MHz}, \mathbf{d m s o}-\boldsymbol{d}_{\boldsymbol{\sigma}}\right) \boldsymbol{\delta} 10.01$ (s, Me 1H) $9.46(\mathrm{~s}, 1 \mathrm{H}) 8.50(\mathrm{~s}(\mathrm{br}), 1 \mathrm{H}) 7.63(\mathrm{~d}, J=7.2 \mathrm{~Hz}, 1 \mathrm{H}) 7.30(\mathrm{~m}, 8 \mathrm{H}) 4.76$ $(\mathrm{d}, J=5.6 \mathrm{~Hz}, 2 \mathrm{H}) 2.38(\mathrm{~s}, 3 \mathrm{H}) .{ }^{13} \mathbf{C}$ NMR (100 MHz, dmso-d $\left.\boldsymbol{d}_{\boldsymbol{6}}\right) \delta 168.6$ 130.6130 .1128 .0127 .0126 .6125 .346 .8 19.7. HPLC Retention time: 7.23 min. m.p. 197.8 - $198.6{ }^{\circ} \mathrm{C}$. IR 3366.0 (s) 3204.0 (s) 3028.4 (m) 2913.3 (w) 1670.1 (s) 1542.3 (s) 1451.2 (m) 1338.1 (w) 1264.9 (m) 1223.4 (s) 1154.8 (m) 1117.7 (m). HRMS Calcd $\mathrm{C}_{16} \mathrm{H}_{17} \mathrm{~N}_{3} \mathrm{OS}$ : 300.1171. Found: 300.1175.

$N$-Benzyl-(5-(2-methylphenyl)-[1,3,4]oxadiazol-2-yl)-amine (12): ${ }^{1} \mathrm{H}$ NMR (400 MHz, dmso$\overbrace{O}^{\mathrm{Me}} \mathrm{N}_{\mathrm{H}}^{\mathrm{N}}$ $\left.\boldsymbol{d}_{\boldsymbol{6}}\right) \delta 8.32(\mathrm{t}, J=6.0 \mathrm{~Hz}, 1 \mathrm{H}) 7.69(\mathrm{~d}, J=7.6 \mathrm{z}, 1 \mathrm{H}) 7.35(\mathrm{~m}, 7 \mathrm{H}) 7.26(\mathrm{t}, J=$ 
7.2Hz, 1H) $4.44(\mathrm{~d}, J=6.0 \mathrm{~Hz}, 2 \mathrm{H}) 2.54(\mathrm{~s}, 9 \mathrm{H}) .{ }^{13} \mathbf{C}$ NMR (100 MHz, dmso-d $) \delta 163.2157 .8$ 138.8 136.4 131.5 130.0 128.3 127.4 127.1 126.3 123.2 46.1 21.5. HPLC Retention time: 9.32 min. m.p. 162.4 - $164.4{ }^{\circ} \mathrm{C}$. IR 3063.4 (w) 2896.7 (s) 1713.3 (s) 1614.2 (m) 1594.2 (w) 1455.8 (m) 1274.8 (m) 1036.2 (m). HRMS Calcd $\mathrm{C}_{16} \mathrm{H}_{15} \mathrm{~N}_{3} \mathrm{O}: 266.1293$. Found: 266.1286.

$\boldsymbol{N}$-Benzyl-iso-nictonic acid semicarbazide: ${ }^{1} \mathrm{H}$ NMR (400 MHz, dmso-d $\left.{ }_{6}\right) \delta 10.48$ (s, $\left.1 \mathrm{H}\right) 8.74(\mathrm{~d}$,<smiles>O=C(NCc1ccccc1)NNC(=O)c1ccncc1</smiles>
$J=4.4 \mathrm{~Hz}, 2 \mathrm{H}) 8.12(\mathrm{~s}, 1 \mathrm{H}) 7.80(\mathrm{~d}, J=4.8 \mathrm{~Hz}, 2 \mathrm{H}) 7.28(\mathrm{~m}, 5 \mathrm{H}) 7.20(\mathrm{t}, J=$ $6.8 \mathrm{~Hz}, 1 \mathrm{H}) 4.24(\mathrm{~d}, J=6.0 \mathrm{~Hz}, 2 \mathrm{H}) .{ }^{13} \mathbf{C}$ NMR (100 MHz, dmso-d $\left.)\right) \delta 164.9$ 158.2 150.2 140.5 139.8 128.1 126.9126 .5121 .5 42.6. HPLC Retention time: 1.58 min. m.p. 215.7 - $217.6^{\circ} \mathrm{C}$. IR 3273.6 (s) 3084.7 (s) 2932.3 (s) 1652.8 (s) 1539.0 (s) 1408.2 (s) 1235.5 (s) 1064.2 (s). HRMS Calcd $\mathrm{C}_{14} \mathrm{H}_{14} \mathrm{~N}_{4} \mathrm{O}_{2}: 271.1184$. Found: 271.1187.

$\boldsymbol{N}$-Benzyl-iso-nictonic acid thio-semicarbazide: ${ }^{\mathbf{1}} \mathrm{H}$ NMR (400 MHz, dmso- $\left.\boldsymbol{d}_{\boldsymbol{\sigma}}\right) \boldsymbol{\delta} 10.72(\mathrm{~s}, 1 \mathrm{H})$ (1) $9.57(\mathrm{~s}, 1 \mathrm{H}) 8.75(\mathrm{~d}, J=4.8 \mathrm{~Hz}, 2 \mathrm{H}) 8.74(\mathrm{~s}, 1 \mathrm{H}) 7.83(\mathrm{~d}, J=4.8 \mathrm{~Hz}, 2 \mathrm{H})$ $7.20(\mathrm{~m}, 5 \mathrm{H}) 4.74(\mathrm{~d}, J=5.6 \mathrm{~Hz}, 2 \mathrm{H}) .{ }^{13} \mathrm{C}$ NMR (100 MHz, dmso-d $\left.)\right) \delta$

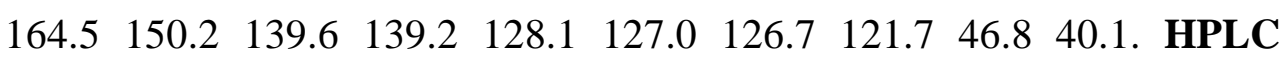
Retention time: 2.58 min. m.p. 244.9 - $246.8{ }^{\circ} \mathrm{C}$. IR 3276.2 (s) 2935.5 (s) 1675.0 (s) 1506.9 (s) 1324.8 (s) 1266.6 (s) 1061.6 (s) 1028.2 (s). HRMS Calcd $\mathrm{C}_{14} \mathrm{H}_{14} \mathrm{~N}_{4} \mathrm{OS}$ : 287.0967. Found: 287.0961.

$N$-Benzyl-(5-pyridin-4-yl-[1,3,4]oxadiazol-2-yl)-amine (13): ${ }^{1} \mathrm{H}$ NMR (400 MHz, dmso-d 6 ) $\delta$ (1) $8.72(\mathrm{dd}, J=1.6 \mathrm{~Hz}, 4.4 \mathrm{~Hz}, 2 \mathrm{H}) 8.61(\mathrm{t}, J=6.0 \mathrm{~Hz}, 1 \mathrm{H}) 7.71(\mathrm{dd}, J=1.6 \mathrm{~Hz}$, $4.4 \mathrm{~Hz}, 2 \mathrm{H}) 7.36(\mathrm{~m}, 4 \mathrm{H}) 7.26(\mathrm{t}, J=7.2 \mathrm{~Hz}, 1 \mathrm{H}) 4.47(\mathrm{~d}, J=6.4 \mathrm{~Hz}, 2 \mathrm{H}) .{ }^{13} \mathrm{C}$ NMR (100 MHz, dmso-d d $_{\text {) }}^{\delta} 164.2156 .1 \quad 150.7138 .5131 .0128 .4127 .4$ 127.2 118.8 46.0. HPLC Retention time: 2.36 min. m.p. 173.4 - 174.2 ${ }^{\circ}$ C.IR 3227.2 (s) 3032.5(s) 1616.1 (s) 1577.3 (s) 1413.5 (s) 1273.0 (m) 1040.3 (s). HRMS Calcd $\mathrm{C}_{14} \mathrm{H}_{12} \mathrm{~N}_{4} \mathrm{O}: 253.1089$. Found: 253.1090 .

$\boldsymbol{N}$-Benzyl-2-thiophenyl acid semicarbazide: ${ }^{1} \mathbf{H}$ NMR (400 MHz, dmso-d $\boldsymbol{~}^{\text {) }} \boldsymbol{\delta} 10.19$ (s, 1H) 8.03<smiles>O=C(NCc1ccccc1)NNC(=O)c1cccs1</smiles>
(s, 1H) $7.82(\mathrm{~m}, 2 \mathrm{H}) 7.22(\mathrm{~m}, 6 \mathrm{H}) 7.09$ (s (br), 1H) $4.23(\mathrm{~d}, J=6.0 \mathrm{~Hz}, 2 \mathrm{H})$. ${ }^{13}$ C NMR (100 MHz, dmso-d d $\delta 161.5158 .4140 .6137 .7 \quad 131.4128 .9$ 
128.1 128.0 126.9 126.5 42.6. HPLC Retention time: 3.57 min. m.p. 184.5 - $186.0{ }^{\circ}$ C. IR 3321.5 (s) 3030.8 (m) 1653.1 (s) 1566.1 (s) 1495.9 (s) 1356.3 (s) 1245.8 (s) 1059.4 (m). HRMS Calcd $\mathrm{C}_{13} \mathrm{H}_{13} \mathrm{~N}_{3} \mathrm{O}_{2} \mathrm{~S}$ : 276.0807. Found: 276.0801 .

$\boldsymbol{N}$-Benzyl-2-thiophenyl acid thio-semicarbazide: ${ }^{1} \mathrm{H}$ NMR (400 MHz, dmso- $\left.\boldsymbol{d}_{\boldsymbol{\sigma}}\right) \boldsymbol{\delta} 10.4(\mathrm{~s}, 1 \mathrm{H})$ II $9.48(\mathrm{~s}, 1 \mathrm{H}) 8.72(\mathrm{~s}(\mathrm{br}), 1 \mathrm{H}) 7.83(\mathrm{~d}, J=4.8 \mathrm{~Hz}, 2 \mathrm{H}) 7.29(\mathrm{~m}, 4 \mathrm{H}) 7.20(\mathrm{~m}$, 2H) $4.72(\mathrm{~d}, J=5.6 \mathrm{~Hz}, 2 \mathrm{H}) .{ }^{13} \mathrm{C}$ NMR (100 MHz, dmso-d $)_{6} \delta 161.1137 .5$ 131.7129 .5128 .0127 .1126 .6 67.0 46.7 25.1 HPLC Retention time: 5.77 min. m.p. 202.5 - $203.8^{\circ} \mathrm{C}$. IR 3155.2 (s) 2974.6 (s) 1676.0 (s) 1558.3 (s) 1493.3 (s) 1353.6 (s) 1272.4 (s) 1235.8 (s) 1069.8 (s). HRMS Calcd $\mathrm{C}_{13} \mathrm{H}_{13} \mathrm{~N}_{3} \mathrm{OS}_{2}$ : 292.0578. Found: 292.0580.

$N$-Benzyl-(5-thiophen-2-yl-[1,3,4]oxadiazol-2-yl)-amine (14): ${ }^{1} \mathrm{H}$ NMR (400 MHz, dmso- $\left.d_{6}\right) \delta$ II $8.36(\mathrm{t}, J=6.0 \mathrm{~Hz}, 1 \mathrm{H}) 7.76(\mathrm{~d}, J=5.2 \mathrm{~Hz}, 1 \mathrm{H})(\mathrm{m}, 1 \mathrm{H}) 7.35(\mathrm{~m}, 4 \mathrm{H}) 7.26(\mathrm{~m}$, 1H) $7.20(\mathrm{~m}, 1 \mathrm{H}) 4.42(\mathrm{~d}, J=6.0 \mathrm{~Hz}, 2 \mathrm{H}) .{ }^{13} \mathbf{C}$ NMR (100 MHz, dmso-d $) \delta$ 163.1 154.1 138.6 129.2 128.3127 .5127 .4 127.1 125.4 46.1. HPLC Retention time: 7.22 min. m.p. decomposed at $150{ }^{\circ} \mathrm{C}$. IR 3219.8 (s) 3026.9 (s) 1617.3 (s) 1454.6 (m) 1261.1 (m) 1011.8 (s). HRMS Calcd $\mathrm{C}_{13} \mathrm{H}_{11} \mathrm{~N}_{3} \mathrm{OS}$ : 258.0701. Found: 258.0702.

$\boldsymbol{N}$-tert-Butyl-2-fluoro-benzoic acid semicarbazide: ${ }^{1} \mathrm{H}$ NMR $\left(400 \mathrm{MHz}, \mathrm{dmso-} \boldsymbol{d}_{6}\right) \delta 9.88(\mathrm{~s}, 1 \mathrm{H})$<smiles>CC(C)(C)NC(=O)NNC(=O)c1ccccc1F</smiles>
$7.74(\mathrm{~d}, J=2.4 \mathrm{~Hz}, 1 \mathrm{H}) 7.61(\mathrm{dt}, J=1.6 \mathrm{~Hz}, J=7.6 \mathrm{~Hz}, 1 \mathrm{H}) 7.54(\mathrm{~m}, 1 \mathrm{H})$ $7.30(\mathrm{~m}, 2 \mathrm{H}) 5.98(\mathrm{~s}, 1 \mathrm{H}) 1.25(\mathrm{~s}, 9 \mathrm{H}) .{ }^{13} \mathrm{C}$ NMR (100 MHz, dmso-d $\left.)\right) \delta$ $163.7160 .5126 .7132 .8130 .1124 .5122 .4(\mathrm{~d}, J=14.5 \mathrm{~Hz}) 116.2(\mathrm{~d}, J=$ 22.0Hz,) 49.5 29.0. ${ }^{19}$ F NMR (375 MHz, dmso-d $\left.\boldsymbol{d}_{\boldsymbol{\sigma}}\right) \boldsymbol{\delta}$ - 115.0. HPLC Retention time: $3.58 \mathrm{~min}$. m.p. 178.3 - $179.9^{\circ} \mathrm{C} . \mathbf{I R} 3307.1$ (s) 2968.0 (s) 1644.9 (s) 1572.1 (s) 1477.6 (s) 1365.0 (s) 1279.1 (s) 1094.7 (s). HRMS Calcd $\mathrm{C}_{12} \mathrm{H}_{16} \mathrm{FN}_{3} \mathrm{O}_{2}$ : 254.1305. Found: 254.1307.

$\boldsymbol{N}$-tert-Butyl-2-fluoro-benzoic acid thio-semicarbazide: ${ }^{1} \mathrm{H}$ NMR (400 MHz, dmso- $\left.\boldsymbol{d}_{\boldsymbol{\sigma}}\right) \boldsymbol{\delta} 10.26(\mathrm{~s}$,<smiles>CC(C)(C)NC(=S)NNC(=O)c1ccccc1F</smiles>
1H) $9.34(\mathrm{~s}, 1 \mathrm{H}) 7.69(\mathrm{t}, J=6.4 \mathrm{~Hz}, 1 \mathrm{H}) 7.59(\mathrm{~m}, 1 \mathrm{H}) 7.31(\mathrm{~m}, 2 \mathrm{H}) 1.45(\mathrm{~s}$, 9H). ${ }^{13}$ C NMR (100 MHz, dmso-d 6 ) $\delta 158.1133 .2130 .2124 .7122 .0$ (d, $J$ $=14.5 \mathrm{~Hz}) 116.2(\mathrm{~d}, J=21.7 \mathrm{~Hz}) 52.828 .3 .{ }^{19}$ F NMR (375 MHz, dmso-d $\left.)\right)$ $\delta$ - 115.1. HPLC Retention time: 6.52 min. m.p. 127.0 - $128.9{ }^{\circ} \mathrm{C}$. IR 3328.4 (s) 3189.9 (s) 1645.4 
(s) 1539.7 (s) 1362.7 (s) 1273.6 (s) 1096.1 (s). HRMS Calcd $\mathrm{C}_{12} \mathrm{H}_{16} \mathrm{FN}_{3} \mathrm{OS}$ : 270.1076. Found: 270.1073 .

N-tert-Butyl-(5-(2-fluorophenyl)-[1,3,4]oxadiazol-2-yl)-amine-HCl (15): ${ }^{1} \mathrm{H}$ NMR (400 MHz,<smiles>CC(C)(C)NC(=O)NC(C)(C)C</smiles>
dmso-d $\left._{6}\right) \delta 7.83(\mathrm{t}, J=6.8 \mathrm{~Hz}, 1 \mathrm{H}) 7.71(\mathrm{~s}, 1 \mathrm{H}) 7.55(\mathrm{~m}, 1 \mathrm{H}) 7.37(\mathrm{~m}, 2 \mathrm{H}) 1.36$ (s, 9H). ${ }^{13} \mathbf{C}$ NMR (100 MHz, dmso-d $\left.\boldsymbol{d}_{6}\right) \delta 162.4159 .8157 .2132 .5$ (d, $J=$ $8.4 \mathrm{~Hz}) 128.4125 .1(\mathrm{~d}, J=3.3 \mathrm{~Hz}) 116.9(\mathrm{~d}, J=20.7 \mathrm{~Hz}) 112.5(\mathrm{~d}, J=12.0 \mathrm{~Hz})$

51.1 28.3. ${ }^{19}$ F NMR (375 MHz, dmso- $\boldsymbol{d}_{\boldsymbol{\sigma}}$ ) $\boldsymbol{\delta}$ - 113.6. HPLC Retention time: 7.20 min. m.p. 171.8 $173.9^{\circ} \mathrm{C}$. IR 3217.3 (m) 3097.3 (s) 2982.4 (s) 1700.0 (s) 1628.7 (s) 1577.7 (m) 1387.2 (s) 1234.6 (s) 1096.7 (s). HRMS Calcd $\mathrm{C}_{12} \mathrm{H}_{14} \mathrm{FN}_{3} \mathrm{O}$ : 236.1199. Found: 236.1192.

$\boldsymbol{N}$-tert-Butyl-2-methyl-benzoic acid semicarbazide: ${ }^{1} \mathrm{H}$ NMR (400 MHz, dmso- $\left.\boldsymbol{d}_{\boldsymbol{\sigma}}\right) \boldsymbol{\delta} 9.71(\mathrm{~s}, 1 \mathrm{H})$ $\underbrace{M e}_{H \rightarrow H}$ $7.64(\mathrm{~s}, 1 \mathrm{H}) 7.35(\mathrm{~m}, 2 \mathrm{H}) 7.24(\mathrm{~m}, 2 \mathrm{H}) 5.98(\mathrm{~s}, 1 \mathrm{H}) 2.36(\mathrm{~s}, 3 \mathrm{H}) 1.26(\mathrm{~s}$, 9H). ${ }^{13}$ C NMR (100 MHz, dmso-d d $^{\text {) } \delta 168.8} 157.1135 .9135 .0130 .4129 .7$ 127.5 125.4 49.5 29.1 19.3. HPLC Retention time: 3.91 min. m.p. 188.1 $189.8^{\circ} \mathrm{C} . \mathbf{I R} 3374.0$ (s) 3275.1 (s) 2968.1 (s) 1700.2 (s) 1652.6 (s) 1558.0 (s) 1364.1 (s) 1271.3 (s) 1084.1 (s). HRMS Calcd $\mathrm{C}_{13} \mathrm{H}_{19} \mathrm{~N}_{3} \mathrm{O}_{2}: 250.1556$. Found: 250.1561.

$\boldsymbol{N}$-tert-Butyl-2-methyl-benzoic acid thio-semicarbazide: ${ }^{1} \mathrm{H}$ NMR $\left(400 \mathrm{MHz}, \mathbf{d m s o}-\boldsymbol{d}_{\boldsymbol{\sigma}}\right) \boldsymbol{\delta} 10.02$<smiles>Cc1ccccc1C(=O)NNC(=S)NC(C)(C)C</smiles>
(s, 1H) $9.15(\mathrm{~s}, 1 \mathrm{H}) 7.48(\mathrm{~d}, J=6.4 \mathrm{~Hz}, 1 \mathrm{H}) 7.37(\mathrm{t}, J=7.2 \mathrm{~Hz}, 1 \mathrm{H}) 7.24(\mathrm{~m}$, 2H) 2.38 (s, 3H) 1.47 (s, 9H). ${ }^{13}$ C NMR (100 MHz, dmso-d $) \delta 168.2136 .1$

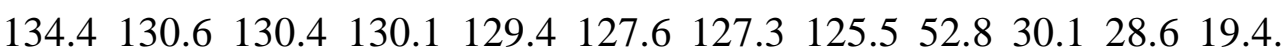
HPLC Retention time: 6.97 min. m.p. 135.4 - $137.4{ }^{\circ}$ C. IR 3273.5 (s) 2965.6 (s) 1672.0 (s) 1525.6 (s) 1361.2 (s) 1268.6 (s) 1176.8 (m) 1077.4 (m). HRMS Calcd $\mathrm{C}_{13} \mathrm{H}_{19} \mathrm{~N}_{3} \mathrm{OS}$ : 266.1327. Found: 266.1332 .

N-tert-Butyl-(5-(2-methylphenyl)-[1,3,4]oxadiazol-2-yl)-amine·HCl (16): ${ }^{1} \mathrm{H}$ NMR (400 $\mathrm{MHz}$, $\mathrm{C}_{\mathrm{N}}^{\mathrm{Me}}$ dmso-d $\left.\boldsymbol{d}_{\boldsymbol{6}}\right) \boldsymbol{\delta} 7.69(\mathrm{t}, J=7.2 \mathrm{~Hz}, 1 \mathrm{H}) 7.68(\mathrm{~s}, 1 \mathrm{H}) 7.35(\mathrm{~m}, 3 \mathrm{H}) 2.57(\mathrm{~s}, 3 \mathrm{H}) 1.37$ (s, 9H). ${ }^{13}$ C NMR (100 MHz, dmso-d $\left.\boldsymbol{d}_{\boldsymbol{\sigma}}\right) \delta 161.8157 .3136 .4131 .5129 .9$ 127.3 126.2 123.3 51.0 28.3 21.6. HPLC Retention time: 8.65 min. m.p. 162.9 
- $164.5^{\circ} \mathrm{C}$. IR 3224.2 (m) 3109.5 (s) 2979.1 (s) 1700.2 (s) 1615.0 (s) 1472.4 (s) 1277.0 (s) 1091.7 (s). HRMS Calcd $\mathrm{C}_{13} \mathrm{H}_{17} \mathrm{~N}_{3} \mathrm{O}: 232.1450$. Found: 232.1453.

$N$-tert-Butyl-2-methoxy-benzoic acid semicarbazide: ${ }^{1} \mathrm{H}$ NMR $\left(400 \mathrm{MHz}, \mathbf{d m s o}-\boldsymbol{d}_{\boldsymbol{\sigma}}\right) \delta 9.68(\mathrm{~d}, J$<smiles>CC(C)(C)NC(=O)NNC(=O)c1ccccc1O</smiles>
$=3.2 \mathrm{~Hz}, 1 \mathrm{H}) 7.84(\mathrm{~d}, J=3.2 \mathrm{~Hz}, 1 \mathrm{H}) 7.69(\mathrm{dd}, J=1.2 \mathrm{~Hz}, J=7.6 \mathrm{~Hz}, 1 \mathrm{H})$ $7.48(\mathrm{dt}, J=1.6 \mathrm{~Hz}, J=8.8 \mathrm{~Hz}, 1 \mathrm{H}) 7.14(\mathrm{~d}, J=7.6 \mathrm{~Hz}, 1 \mathrm{H}) 7.04(\mathrm{t}, J=$ 7.6Hz, 1H) $6.0(\mathrm{~s}(\mathrm{br}), 1 \mathrm{H}) .{ }^{13} \mathrm{C}$ NMR (100 MHz, dmso-d $\left.\boldsymbol{d}_{6}\right) \delta 164.5156 .9$ 156.7132 .5130 .3121 .8120 .5112 .055 .949 .4 29.1. HPLC Retention time: 4.18 min. m.p. 140.6 $142.0{ }^{\circ} \mathrm{C}$. IR 3372.5 (s) 2975.6 (s) 1706.7 (s) 1632.7 (s) 1535.0 (s) 1436.8 (s) 1265.4 (s) 1017.4 (s). HRMS Calcd $\mathrm{C}_{13} \mathrm{H}_{19} \mathrm{~N}_{3} \mathrm{O}_{3}: 266.1505$. Found: 266.1501.

$N$-tert-Butyl-2-methoxy-benzoic acid thio-semicarbazide: ${ }^{1} \mathrm{H}$ NMR (400 MHz, dmso- $\left.d_{6}\right) \delta 10.0$<smiles>COc1ccccc1C(=O)NNC(=S)NC(C)(C)C</smiles>
(s (br), 1H) $7.51(\mathrm{~s}(\mathrm{br}), 1 \mathrm{H}) 7.51(\mathrm{t}, J=7.2 \mathrm{~Hz}, 1 \mathrm{H}) 7.17(\mathrm{~d}, J=8.0 \mathrm{~Hz}, 1 \mathrm{H})$ $7.06(\mathrm{t}, J=7.6 \mathrm{~Hz}, 1 \mathrm{H}) 3.92(\mathrm{~d}, J=8.0 \mathrm{~Hz}, 2 \mathrm{H}) 1.45(\mathrm{~s}, 9 \mathrm{H}) .{ }^{13} \mathbf{C}$ NMR (100 MHz, dmso- $\left.d_{\sigma}\right) \delta 164.0156 .9132 .8130 .6130 .2120 .7112 .156 .152 .7$ 28.6. HPLC Retention time: 7.30 min. m.p. 141.0 - $142.5{ }^{\circ} \mathrm{C}$. IR 3323.3 (s) 3187.5 (s) 2965.5 (s) 1640.9 (s) 1539.8 (s) 1433.3 (m) 1276.3 (s) 1162.4 (m) 1019.0 (s). HRMS Calcd $\mathrm{C}_{13} \mathrm{H}_{19} \mathrm{~N}_{3} \mathrm{O}_{2} \mathrm{~S}$ : 282.1176. Found: 282.1277.

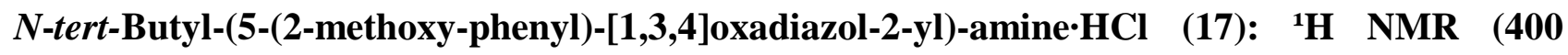
$\underbrace{\mathrm{Me}}_{\mathrm{Me}}$ MHz, dmso- $\left.d_{6}\right): \delta 7.67(\mathrm{~d}, J=7.7 \mathrm{~Hz}, 1 \mathrm{H}) 7.52(\mathrm{~m}, 1 \mathrm{H}) 7.21(\mathrm{~d}, J=8.6 \mathrm{~Hz}, 1$ H) $7.08(\mathrm{t}, J=7.5 \mathrm{~Hz}, 1 \mathrm{H}) 3.85(\mathrm{~s}, 3 \mathrm{H}) 1.37(\mathrm{~s}, 9 \mathrm{H}) .{ }^{13} \mathrm{C}$ NMR $(\mathbf{1 0 0} \mathbf{~ M H z}$, dmso- $\left.\boldsymbol{d}_{\boldsymbol{\sigma}}\right): \boldsymbol{\delta} 160.4,132.9,129.5,120.8,112.6,112.4,56.0,52.0,28.3$. HPLC Retention time: 6.58 min. m.p. 196.8 - $198.3{ }^{\circ} \mathrm{C}$. IR 3060.6 (s) 2841.6 (s) 1684.5 (s) 1598.8 (s) 1487.3 (s) 1372.5 (s) 1269.7 (s) 1183.4 (s) 1075.9 (s). HRMS Calcd $\mathrm{C}_{13} \mathrm{H}_{17} \mathrm{~N}_{3} \mathrm{O}_{2}: 248.1399$. Found: 248.1393.

$N$-Phenyl-4-dimethylamino-benzoic acid semicarbazide: ${ }^{1} \mathrm{H}$ NMR (400 MHz, dmso- $\left.\boldsymbol{d}_{\boldsymbol{\sigma}}\right) \boldsymbol{\delta} 9.92$ 年) (s, 1H) $8.80(\mathrm{~s}, 1 \mathrm{H}) 8.00(\mathrm{~s}, 1 \mathrm{H}) 7.78(\mathrm{~d}, J=8.8 \mathrm{~Hz}, 2 \mathrm{H}) 7.46(\mathrm{~d}, J=$ $8.0 \mathrm{~Hz}, 2 \mathrm{H}) 7.24(\mathrm{t}, J=8.0 \mathrm{~Hz}, 2 \mathrm{H}) 6.94(\mathrm{t}, J=7.6 \mathrm{~Hz}, 1 \mathrm{H}) 6.71(\mathrm{~d}, J$ 
$=8.8 \mathrm{~Hz}, 2 \mathrm{H}) 2.97(\mathrm{~s}, 6 \mathrm{H}) .{ }^{\mathbf{1 3}} \mathbf{C}$ NMR (100 MHz, dmso-d $\left.\boldsymbol{d}_{\boldsymbol{\sigma}}\right) \delta 166.4152 .4139 .8129 .0128 .6121 .8$ 118.9118 .4 110.7. HPLC Retention time: 4.30 min. m.p. 250.8 - $251.9{ }^{\circ} \mathrm{C}$. IR 3268.0 (s) 3040.5 (m) 2910.3 (m) 1653.0 (s) 1554.2 (s) 1374.9 (s) 1262.8 (m) 1136.5 (m) 1084.9 (m). HRMS Calcd $\mathrm{C}_{16} \mathrm{H}_{18} \mathrm{~N}_{4} \mathrm{O}_{2}: 299.1508$. Found: 299.1501 .

$N$-Phenyl-4-dimethylamino-benzoic acid thio-semicarbazide: ${ }^{1} \mathrm{H}$ NMR (400 MHz, dmso- $\left.d_{6}\right) \delta$

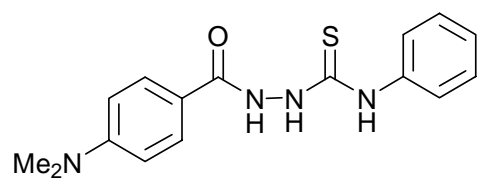
10.15 (s, 1H) 9.72 (br s, 1H) 9.57 (br s, 1H) 7.81 (d, $J=8.8 \mathrm{~Hz}, 2 \mathrm{H})$ $7.45(\mathrm{br}, 2 \mathrm{H}) 7.31(\mathrm{t}, J=8.8 \mathrm{~Hz}, 2 \mathrm{H}) 7.13(\mathrm{t}, J=7.2 \mathrm{~Hz}, 1 \mathrm{H}) 6.72(\mathrm{~d}, J$ $=8.8 \mathrm{~Hz}, 2 \mathrm{H}) 2.98(\mathrm{~s}, 6 \mathrm{H}) .{ }^{13} \mathbf{C}$ NMR $\left(\mathbf{1 0 0} \mathbf{~ M H z}, \mathbf{d m s o}^{-} \boldsymbol{d}_{6}\right) \delta 152.5$

139.3129 .3118 .9 110.6. HPLC Retention time: 5.73 min. m.p. 243.0 - $244.8{ }^{\circ} \mathrm{C}$. IR 3346.8 (s) 3244.5 (s) 1653.7 (s) 1601.4 (s) 1495.7 (s) 1376.4 (s) 1259.6 (s) 1172.4 (s) 1064.2 (m). HRMS Calcd $\mathrm{C}_{16} \mathrm{H}_{18} \mathrm{~N}_{4} \mathrm{OS}: 315.1280$. Found: 315.1288 .

N-tert-Butyl-(5-(4-dimethylamino-phenyl)-[1,3,4]oxadiazol-2-yl)-amine (18): ${ }^{1} \mathrm{H}$ NMR (400<smiles>O=[N+]([O-])c1ccc(-c2nnc(Nc3ccccc3)o2)cc1</smiles>
MHz, dmso-d $\left.d_{6}\right) \delta 10.49(\mathrm{~s}, 1 \mathrm{H}) 7.69(\mathrm{~d}, J=8.4 \mathrm{~Hz}, 2 \mathrm{H}) 7.59(\mathrm{~d}, J=8.0$ $\mathrm{Hz}, 2 \mathrm{H}) 7.34(\mathrm{t}, J=7.7 \mathrm{~Hz}, 2 \mathrm{H}) 6.98(\mathrm{t}, J=7.3 \mathrm{~Hz}, 1 \mathrm{H}) 6.83(\mathrm{~d}, J=$ $8.5 \mathrm{~Hz}, 2 \mathrm{H}) 2.99(\mathrm{~s}, 6 \mathrm{H}) \cdot{ }^{\mathbf{1 3}} \mathbf{C}$ NMR (100 MHz, dmso-d $\left.\boldsymbol{d}_{\boldsymbol{6}}\right) \delta 158.6151 .7$ 129.0126 .8121 .6116 .8 111.9. HPLC Retention time: 9.09 min. m.p. 200.8 - $201.4{ }^{\circ}$ C. IR 3304.3 (m) $2895.1(\mathrm{~m}) 1612.2$ (s) 1567.4 (s) 1450.6 (s) 1369.2 (s) 1236.2 (s) 1172.1 (s) 1067.2 (m). HRMS Calcd $\mathrm{C}_{16} \mathrm{H}_{16} \mathrm{~N}_{4} \mathrm{O}$ : 281.1402. Found: 281.1407 .

$N$-Phenyl-2-methoxy-benzoic acid semicarbazide: ${ }^{1} \mathrm{H}$ NMR (400 MHz, dmso- $\left.\boldsymbol{d}_{\boldsymbol{\sigma}}\right) \delta 9.81$ (s, $\left.1 \mathrm{H}\right)$<smiles>COc1ccccc1C(=O)NNC(=O)Nc1ccccc1</smiles>
$8.74(\mathrm{br} \mathrm{s}, 1 \mathrm{H}) 8.30(\mathrm{~s}, 1 \mathrm{H}) 7.77(\mathrm{dt}, J=1.6 \mathrm{~Hz}, J=8.0 \mathrm{~Hz}, 1 \mathrm{H}) 7.51(\mathrm{dt}, J$ $=1.6 \mathrm{~Hz}, J=8.0 \mathrm{~Hz}, 1 \mathrm{H}) 7.45(\mathrm{~d}, J=7.6 \mathrm{~Hz}, 2 \mathrm{H}) 7.25(\mathrm{t}, J=8.0 \mathrm{~Hz}, 2 \mathrm{H})$ $7.17(\mathrm{~d}, J=8.0 \mathrm{~Hz}, 1 \mathrm{H}) 7.06(\mathrm{t}, J=7.6 \mathrm{~Hz}, 1 \mathrm{H}) 6.95(\mathrm{t}, J=7.6 \mathrm{~Hz}, 1 \mathrm{H}) 3.90$ (s, 3H). ${ }^{13} \mathbf{C}$ NMR (100 MHz, dmso-d $\left.\boldsymbol{d}_{\boldsymbol{\sigma}}\right) \boldsymbol{\delta} 157.1155 .0139 .6132 .8130 .5128 .7121 .9121 .5120 .5$ 118.4 112.1 55.9. HPLC Retention time: 4.80 min. m.p. 234.3 - $234.9^{\circ} \mathrm{C}$. IR 3311.5 (s) 1717.1 (m) 1655.6 (s) 1563.2 (s) 1500.1 (m) 1318.3 (m) 1246.3 (s) 1165.7 (m). HRMS Calcd $\mathrm{C}_{15} \mathrm{H}_{15} \mathrm{~N}_{3} \mathrm{O}_{3}$ : 286.1192. Found: 286.1194. 
$N$-Phenyl-2-methoxy-benzoic acid thio-semicarbazide: ${ }^{1} \mathrm{H}$ NMR $\left(400 \mathrm{MHz}, \mathrm{dmso}-d_{6}\right) \delta 11.00$<smiles>O=C(NNC(=S)Nc1ccccc1)c1ccccc1O</smiles>
(br s, 1H) $10.00-9.55$ (br, 2H) 7.89 (br s, 1H) 7.54 (m, 3H) 7.33 (t, $J=$ $8.0 \mathrm{~Hz}, 2 \mathrm{H}) 7.14$ (m, 3H) 3.92 (s, 3H). ${ }^{13} \mathbf{C}$ NMR (100 MHz, dmso-d $\left.\boldsymbol{d}_{\boldsymbol{\sigma}}\right) \delta$ 157.2139 .1133 .1130 .8128 .3120 .7112 .1 56.2. HPLC Retention time: 6.46 min. m.p. 192.8 - $195.2^{\circ} \mathrm{C}$. IR 3291.7 (s) 2943.1 (m) 1635.6 (s) 1599.5 (s) 1467.5 (s) 1356.4 (s) 1242.9 (s) 1161.4 (s) 1015.8 (s). HRMS Calcd $\mathrm{C}_{15} \mathrm{H}_{15} \mathrm{~N}_{3} \mathrm{O}_{2} \mathrm{~S}: 302.0963$. Found: 302.0968.

$N$-Phenyl-(5-(2-methoxy-phenyl)-[1,3,4]oxadiazol-2-yl)-amine-HCl (19): ${ }^{1} \mathrm{H}$ NMR (400 MHz, (1) dmso-d $\left._{6}\right) \delta 10.72(\mathrm{~s}, 1 \mathrm{H}) 7.74(\mathrm{~d}, J=7.7 \mathrm{~Hz}, 1 \mathrm{H}) 7.62(\mathrm{~d}, J=8.0 \mathrm{~Hz}, 2 \mathrm{H})$ $7.52(\mathrm{~m}, 1 \mathrm{H}) 7.34(\mathrm{t}, J=7.7 \mathrm{~Hz}, 2 \mathrm{H}) 7.22(\mathrm{~d}, J=8.5 \mathrm{~Hz}, 1 \mathrm{H}) 7.09(\mathrm{t}, J=$ $7.6 \mathrm{~Hz}, 1 \mathrm{H}) 6.99(\mathrm{t}, J=7.4 \mathrm{~Hz}, 1 \mathrm{H}) 3.88(\mathrm{~s}, 3 \mathrm{H}) .{ }^{13} \mathbf{C}$ NMR $(\mathbf{1 0 0} \mathbf{~ M H z}$, dmso- $\left.d_{\boldsymbol{\sigma}}\right) \delta 160.0,157.1,138.8,132.8,129.8,129.1,121.9,120.8,117.1,112.8,112.6,56.0$. HPLC Retention time: 7.64 min. m.p. 195.7 - $196.6^{\circ} \mathrm{C}$. IR 3359.6 (m) 3042.4 (m) 1667.0 (s) 1571.6 (s) 1368.6 (m) 1279.3 (s) 1168.1 (s) 1075.4 (s). HRMS Calcd $\mathrm{C}_{15} \mathrm{H}_{13} \mathrm{~N}_{3} \mathrm{O}_{2}: 268.1086$. Found: 268.1089. 


\section{Representative ${ }^{1} \mathrm{H}$ and ${ }^{13} \mathrm{C}$ Spectra:}

N-Benzyl-phenyl acetic acid semicarbazide (1a)::

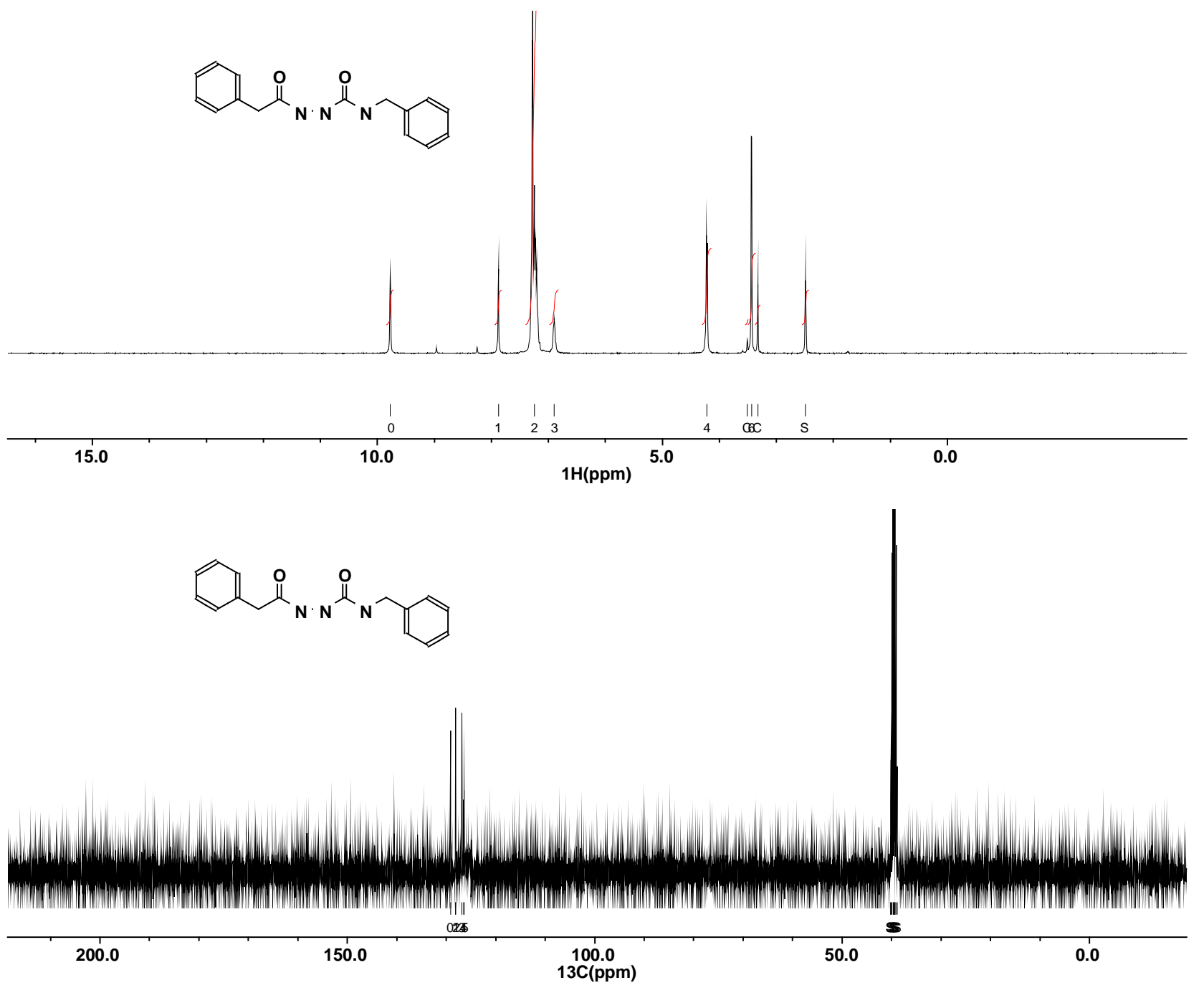


N-Benzyl-phenyl acetic acid thio-semicarbazide (1b):

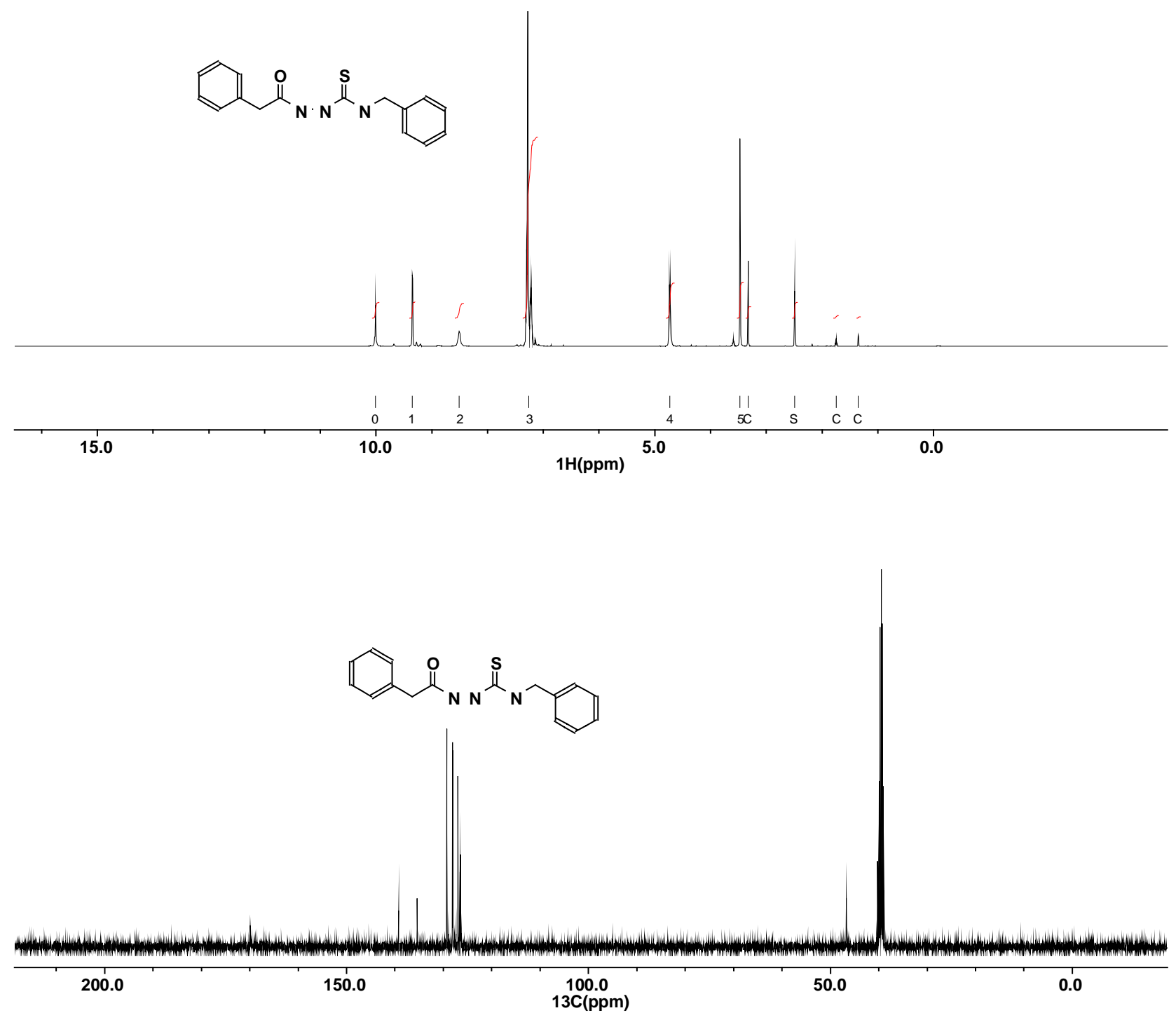


Benzyl-(5-benzyl-[1,3,4]oxadiazol-2-yl)-amine (2):

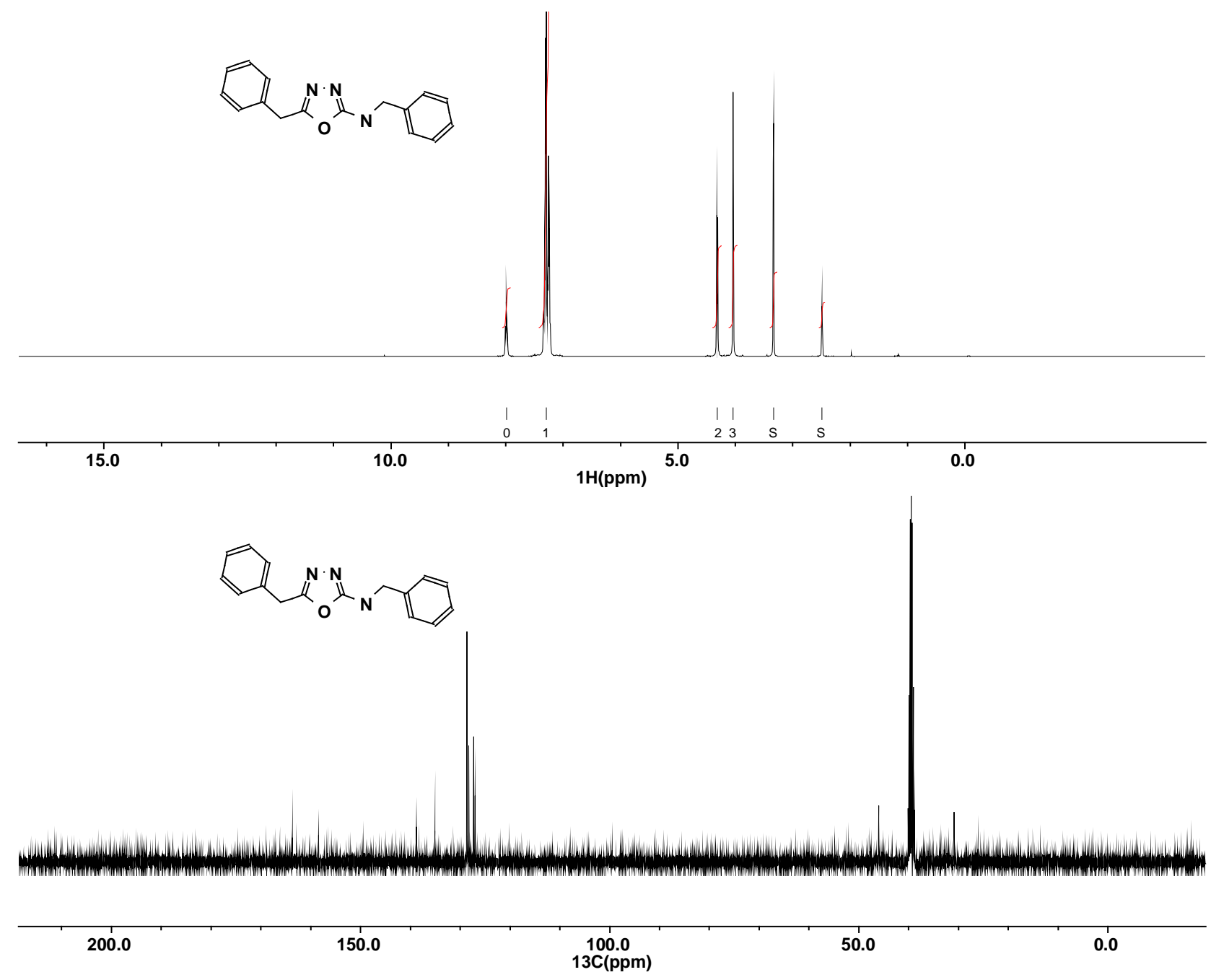


Cyclohexyl-(5-benzyl-[1,3,4]oxadiazol-2-yl)-amine·HCl (3):
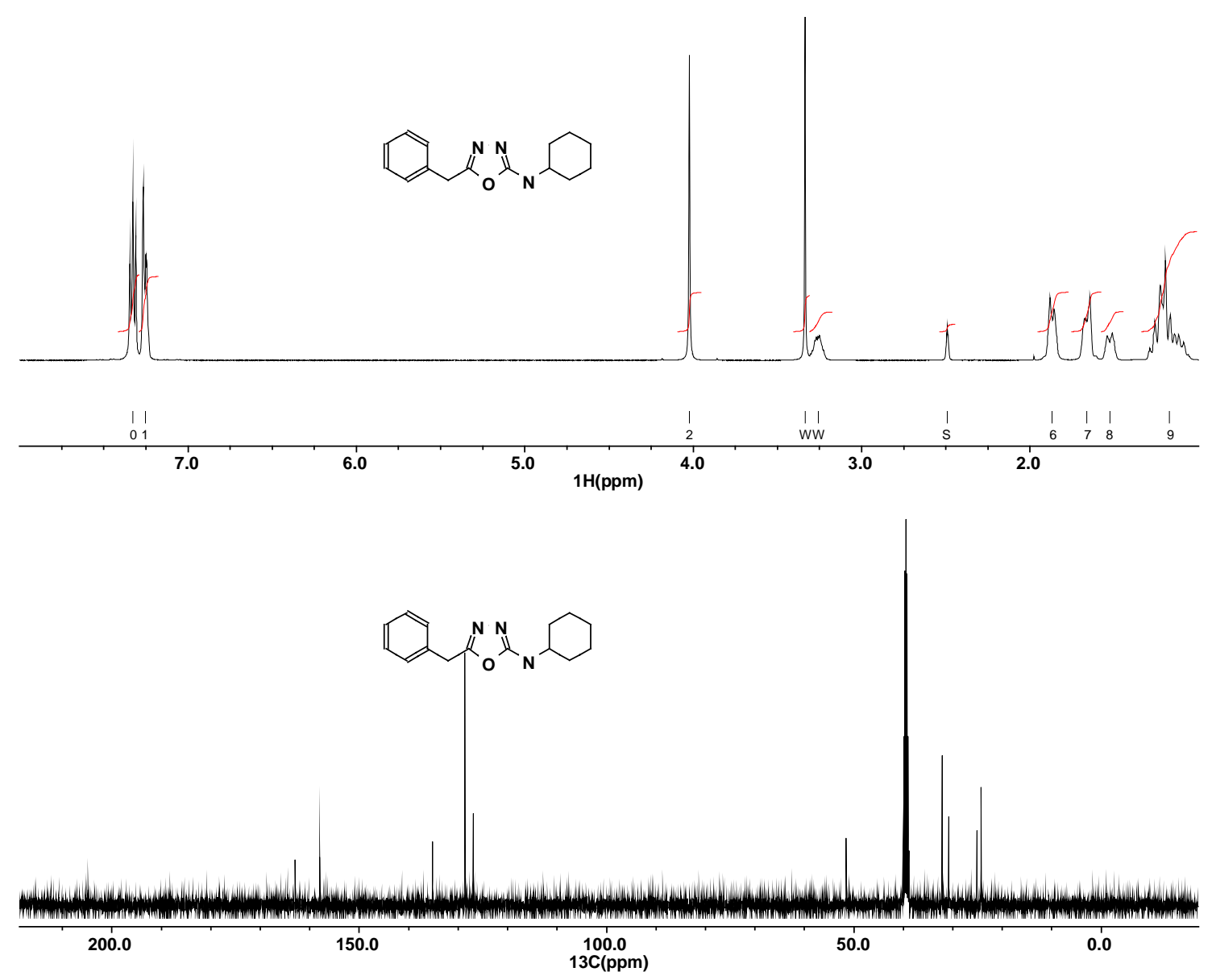
N-tert-Butyl-(5-benzyl-[1,3,4]oxadiazol-2-yl)-amine·HCl (4):

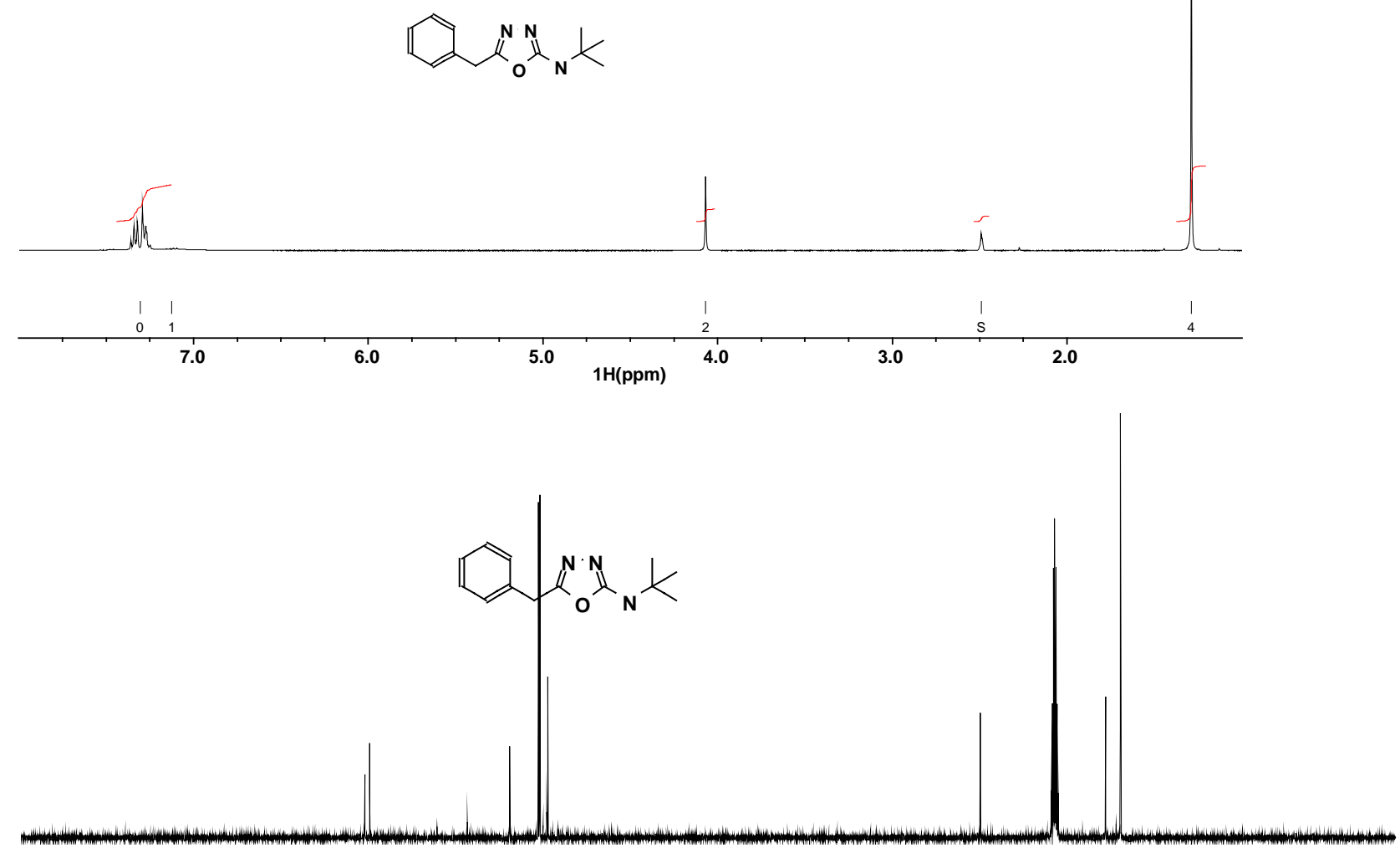

200.0

150.0 
N-Benzyl-(5-butyl-[1,3,4]oxadiazol-2-yl)-amine (5):
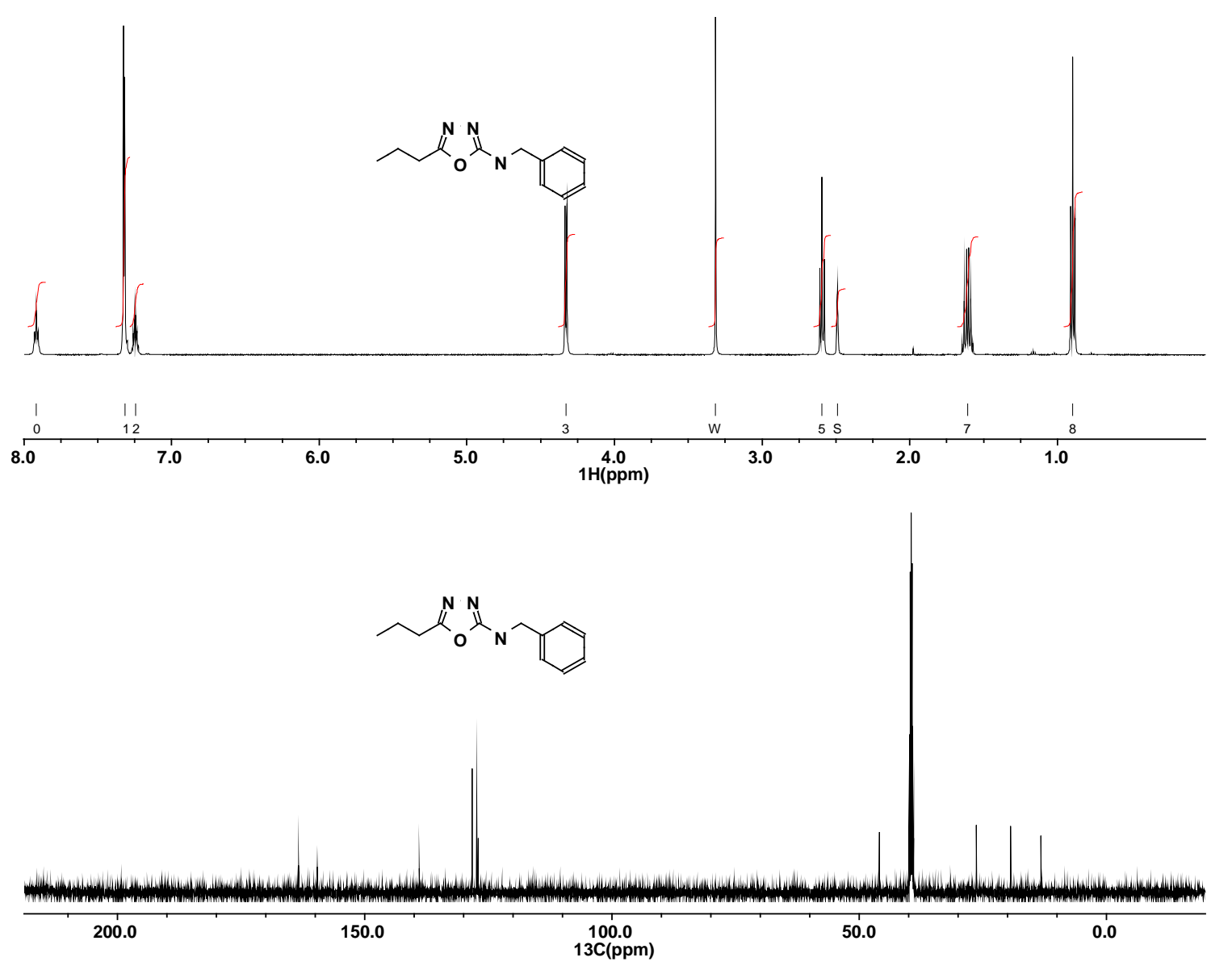
N-Benzyl-(5-(2-hydroxy-2-methyl)-butyl-[1,3,4]oxadiazol-2-yl)-amine-HCl (6):
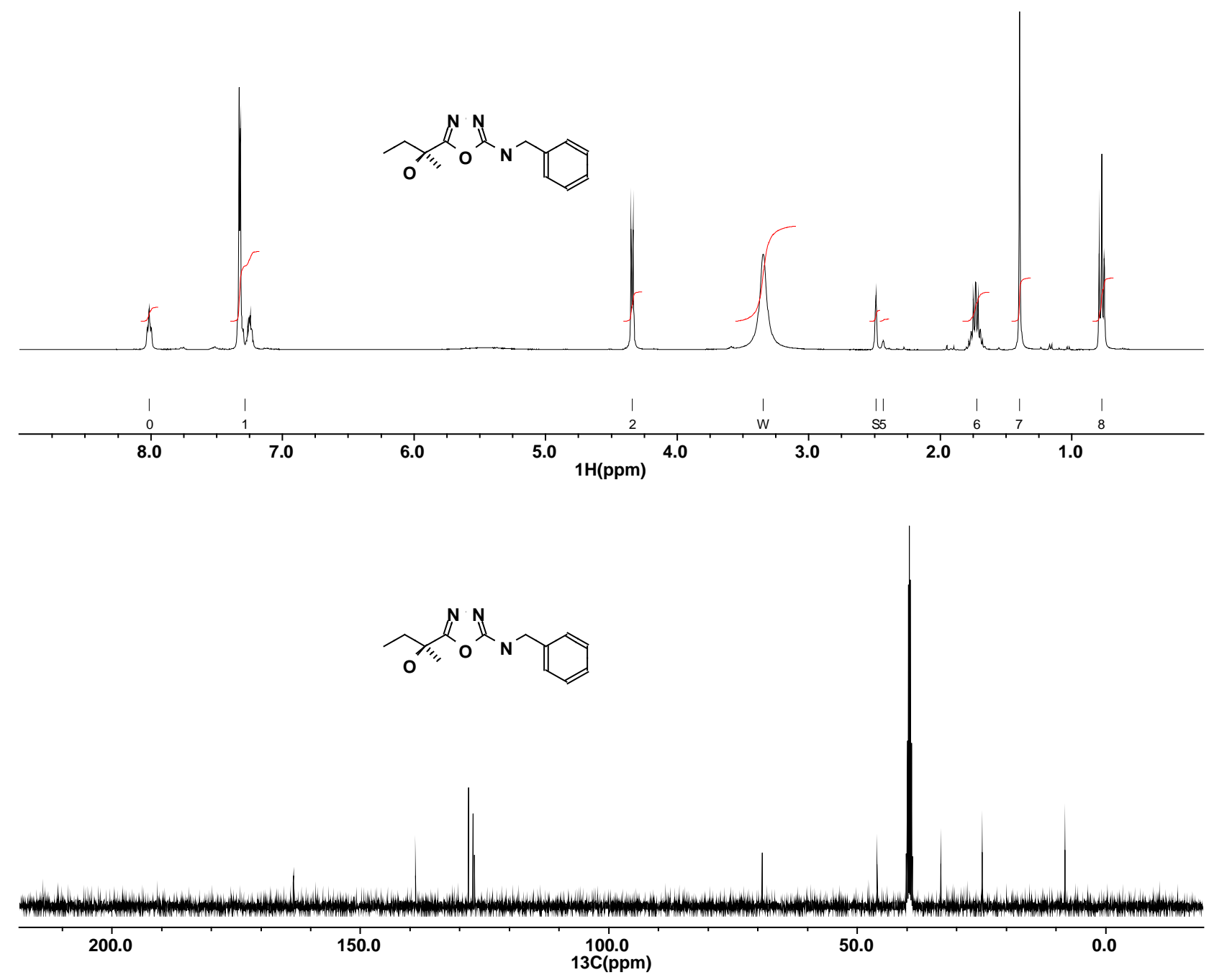
N-Benzyl-(5-tert-butyl-[1,3,4]oxadiazol-2-yl)-amine·HCl (7):<smiles>CC(C)(C)C(=N)OC(=N)NCc1ccccc1</smiles>

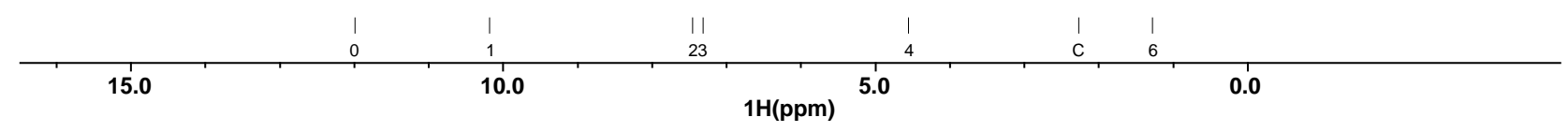
$\gamma_{0}^{N} N_{N}^{N}$

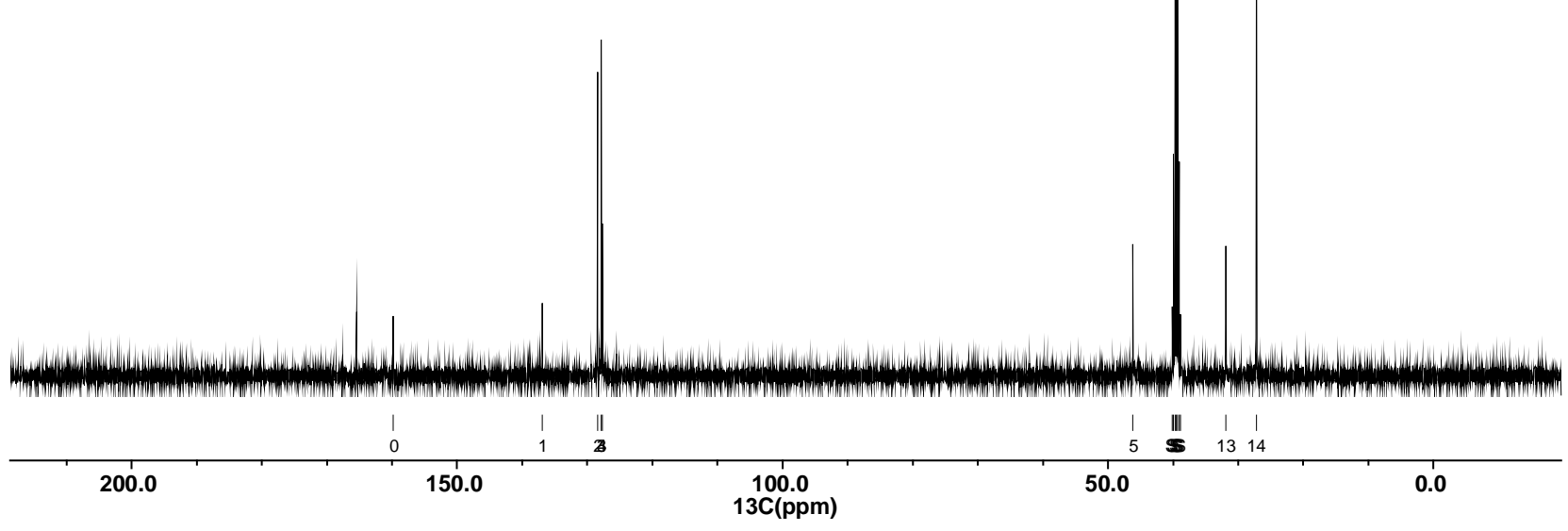


N-Phenyl-(5-benzyl-[1,3,4]oxadiazol-2-yl)-amine·HCl (8):
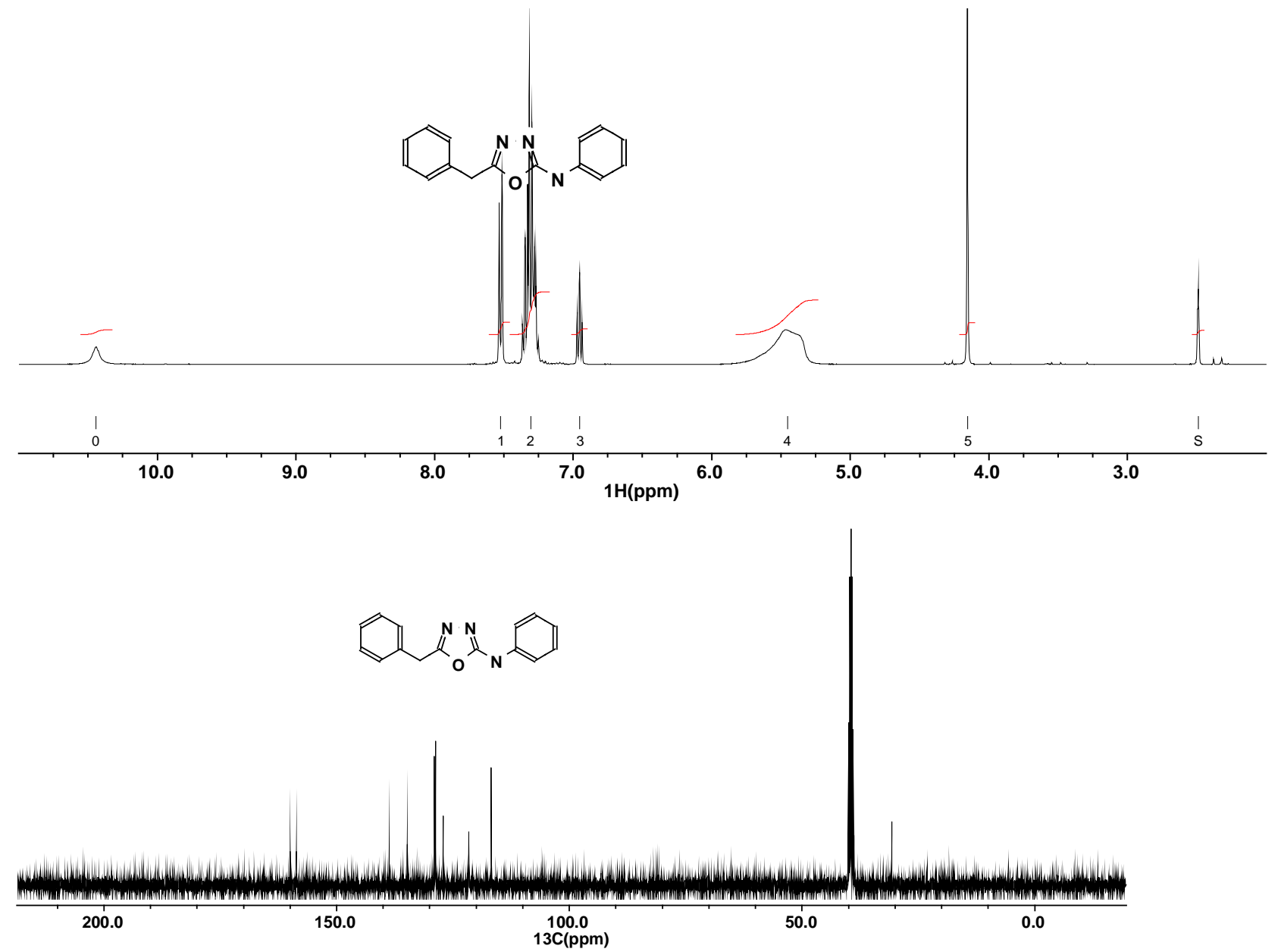
N-Phenyl-(5-methyl-[1,3,4]oxadiazol-2-yl)-amine-HCl (9):

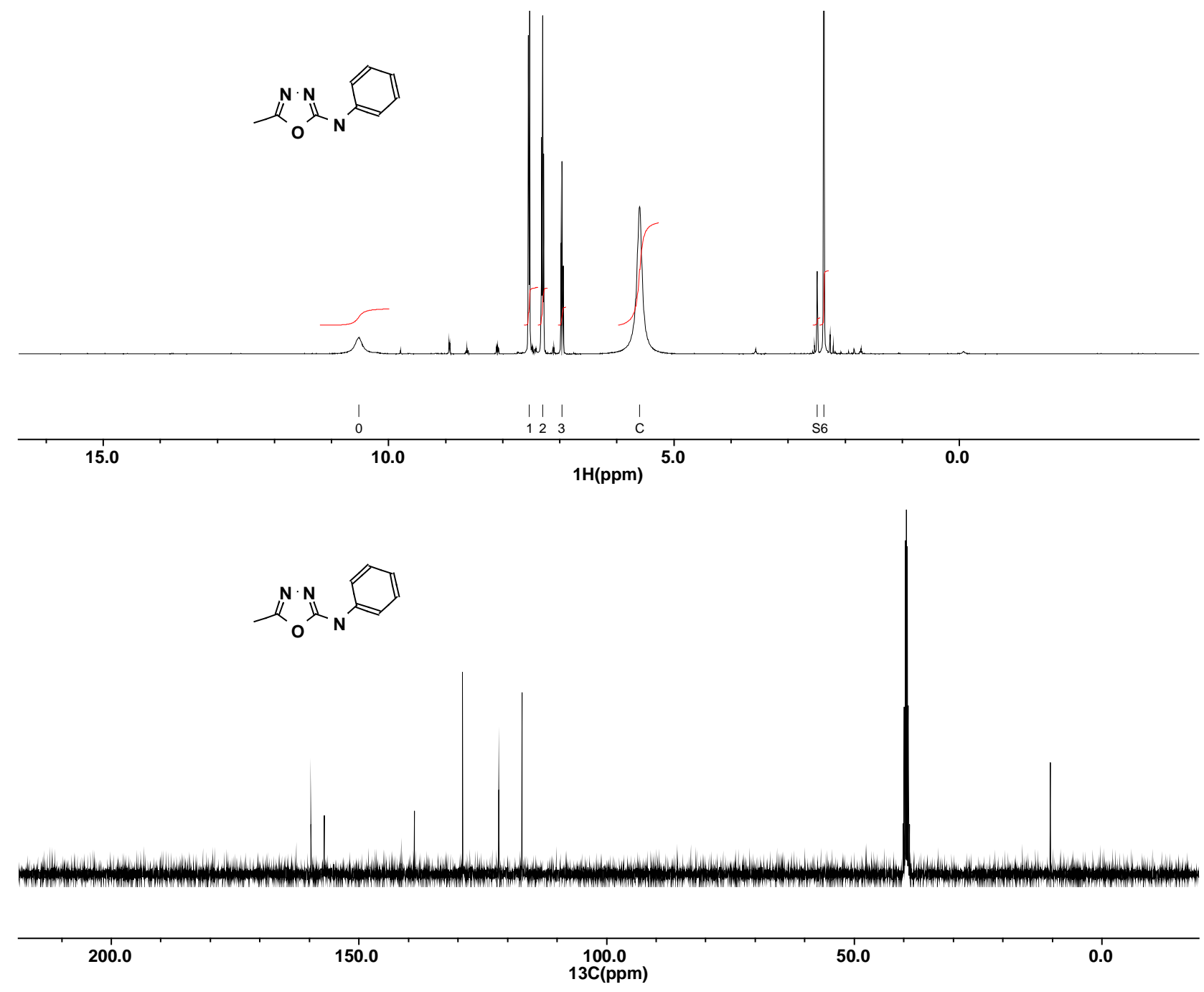


N-Phenyl-(5- trifluoromethyl-[1,3,4]oxadiazol-2-yl)-amine·HCl (10):

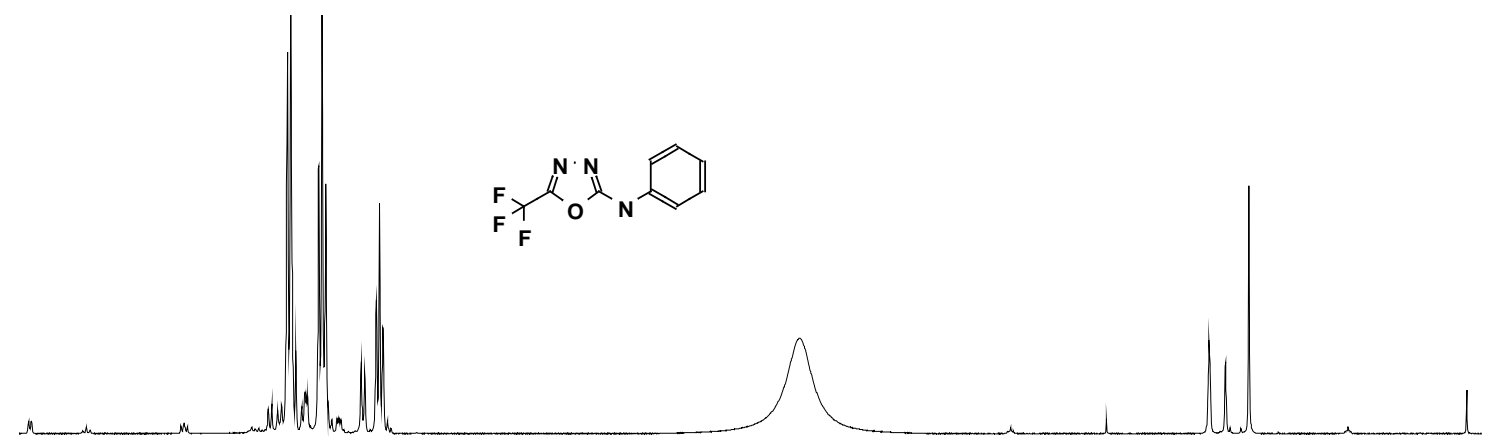

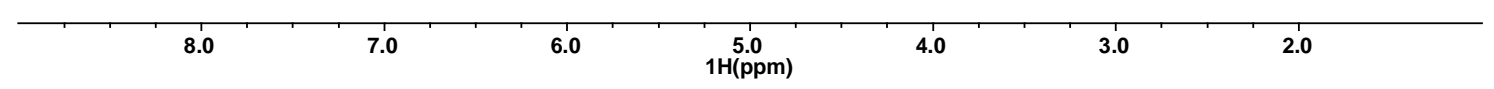

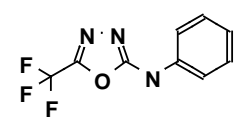

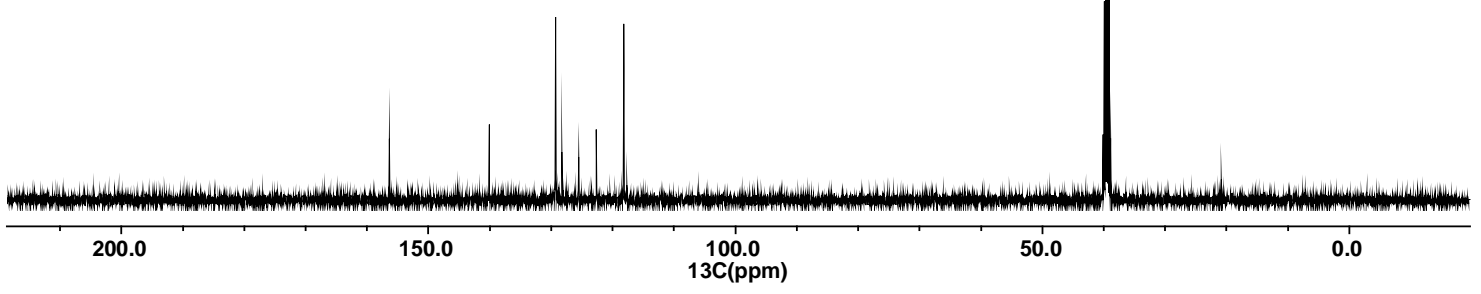


N-Benzyl-(5-(4-fluorophenyl)-[1,3,4]oxadiazol-2-yl)-amine (11):
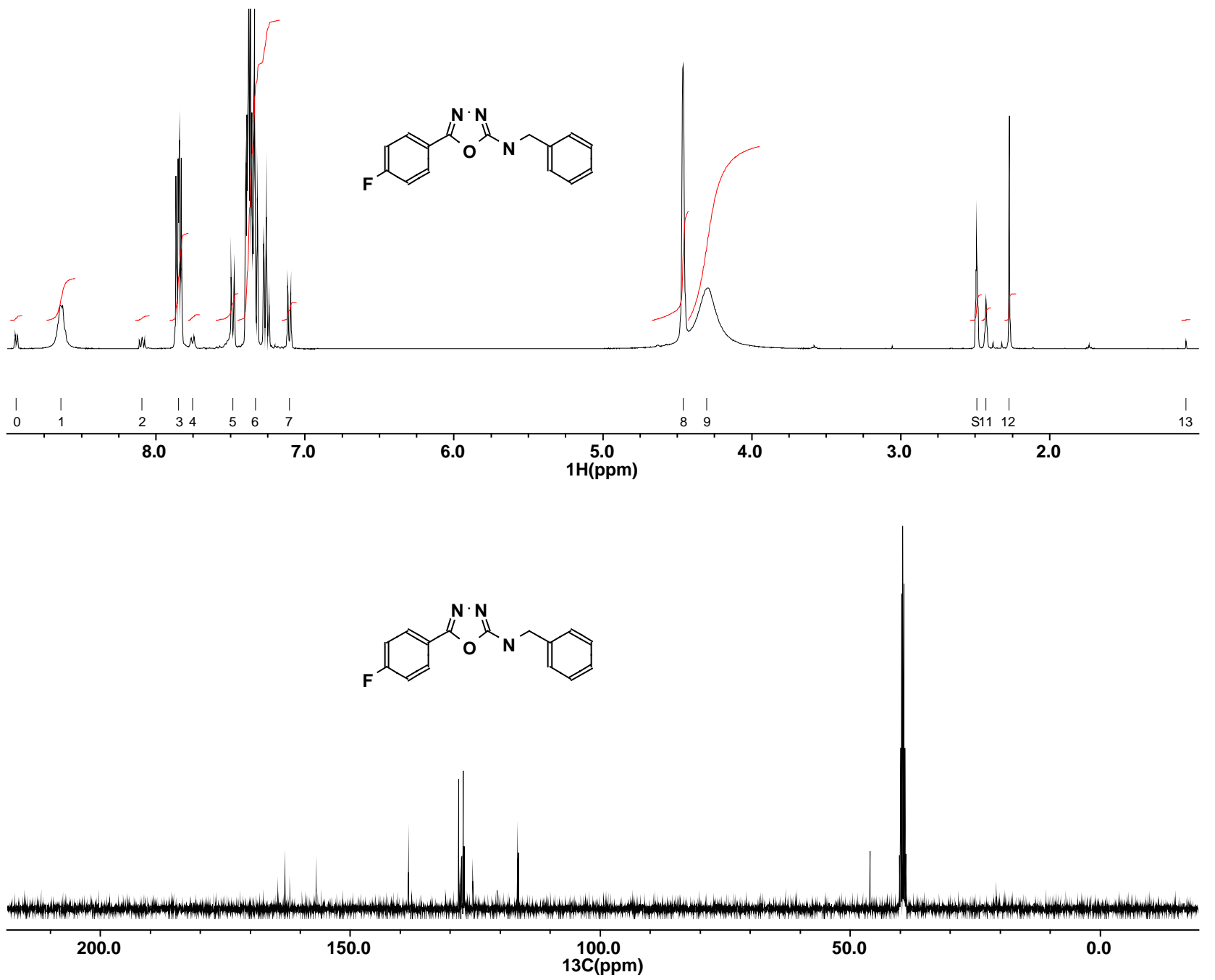
N-Benzyl-(5-(2-methylphenyl)-[1,3,4]oxadiazol-2-yl)-amine (12):
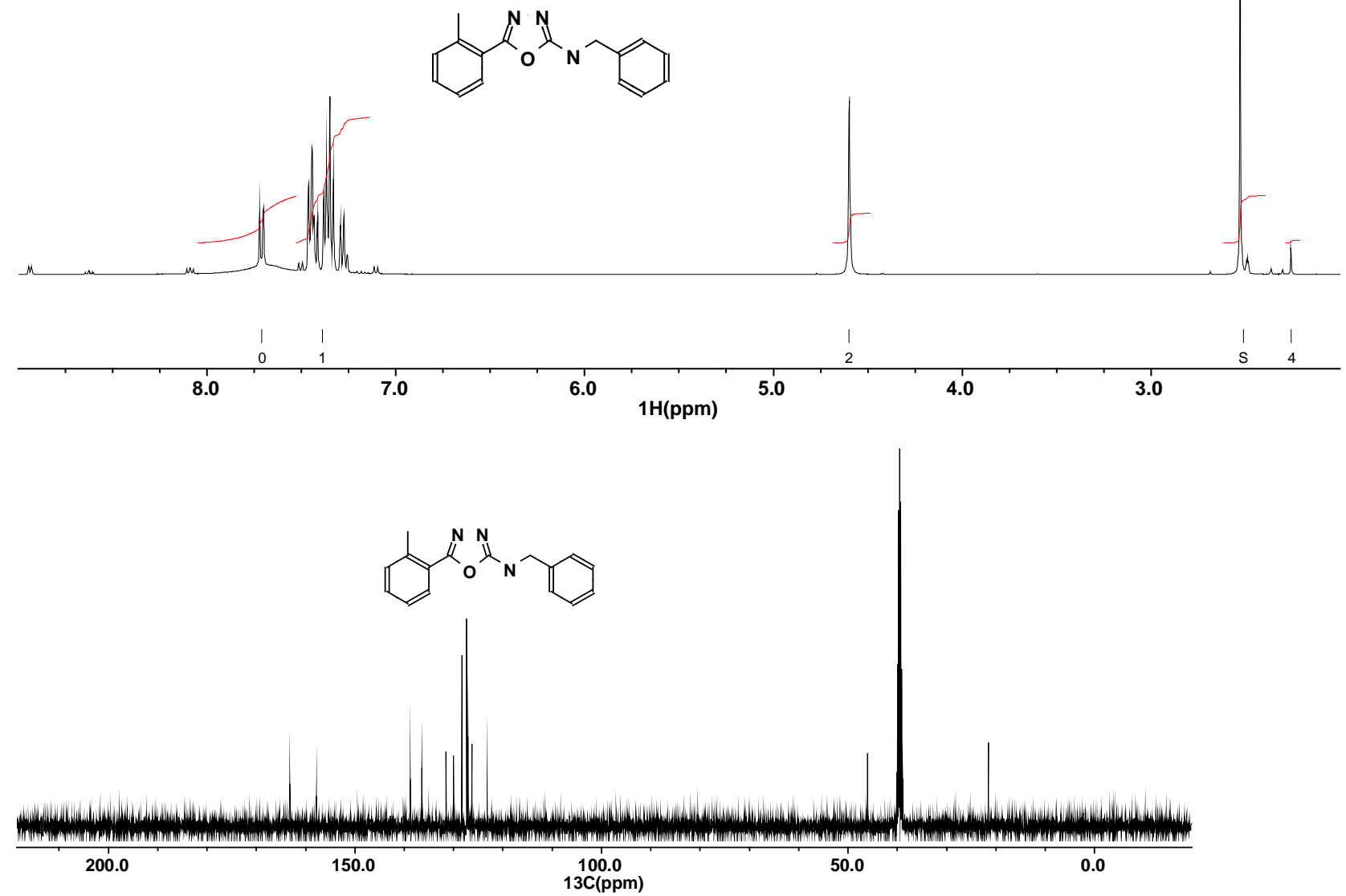
N-Benzyl-(5-pyridin-4-yl-[1,3,4]oxadiazol-2-yl)-amine (13):

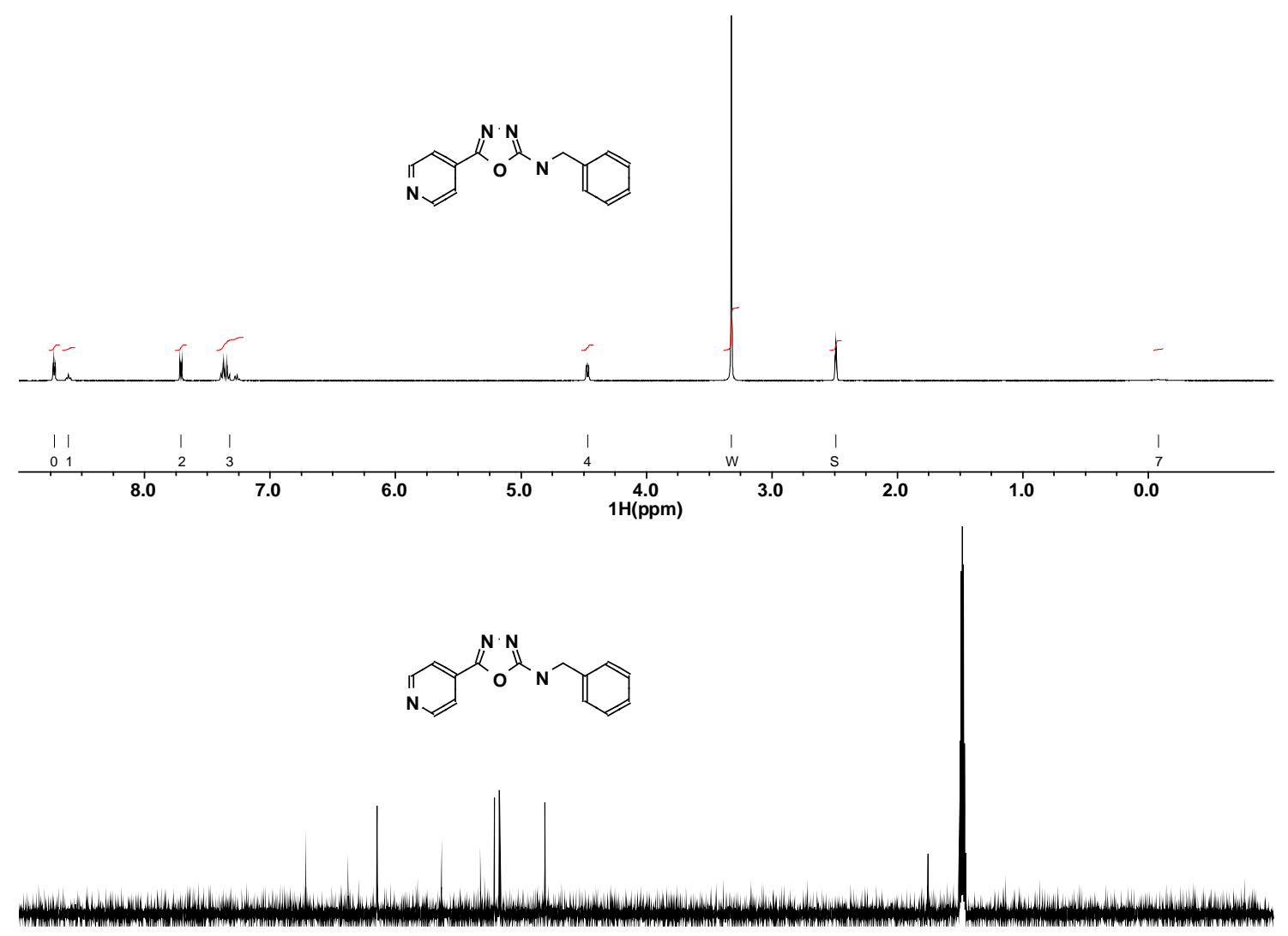


N-Benzyl-(5-thiophen-2-yl-[1,3,4] oxadiazol-2-yl)-amine (14):
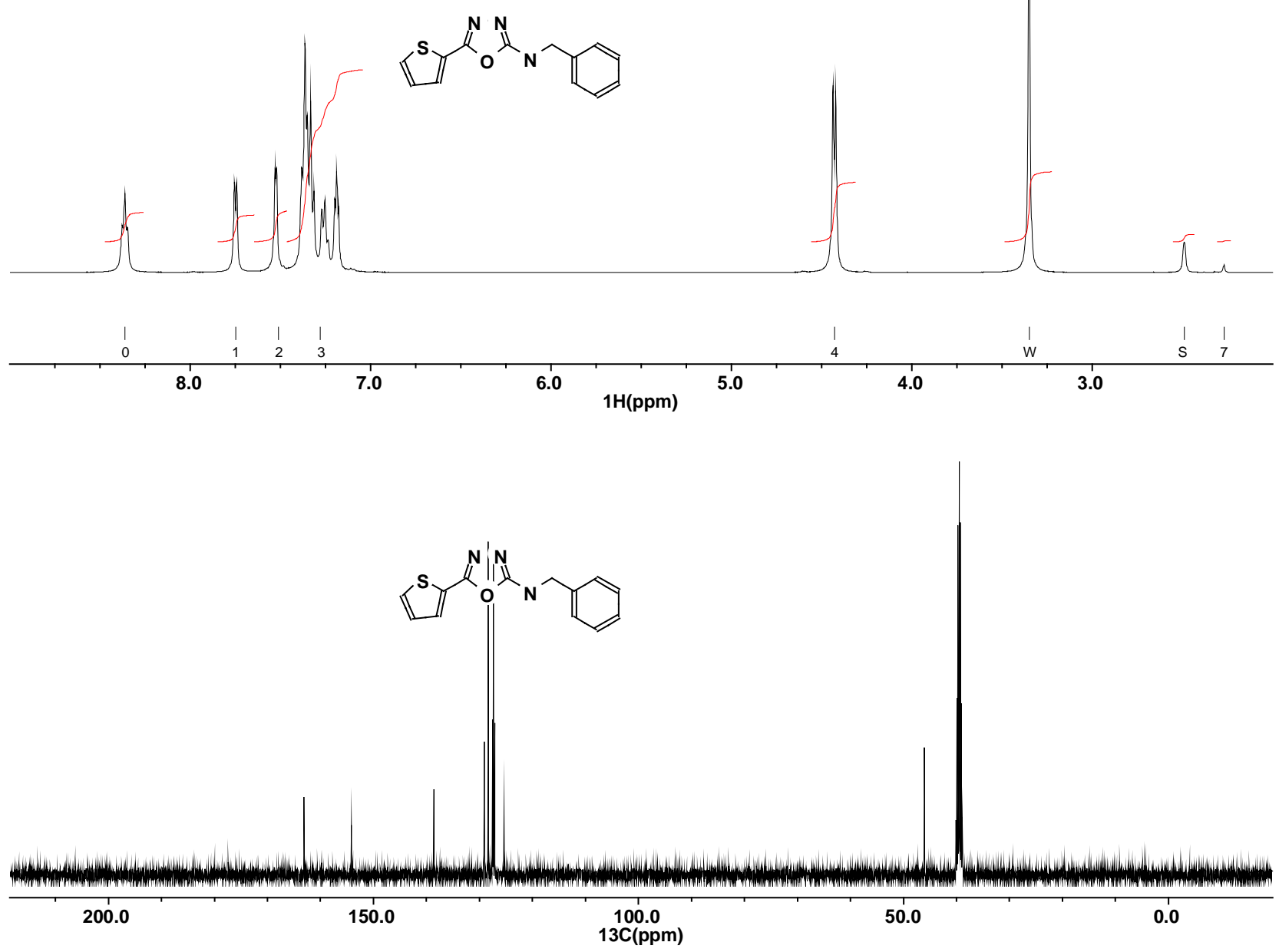
N-tert-Butyl-(5-(2-fluorophenyl)-[1,3,4]oxadiazol-2-yl)-amine·HCl (15):

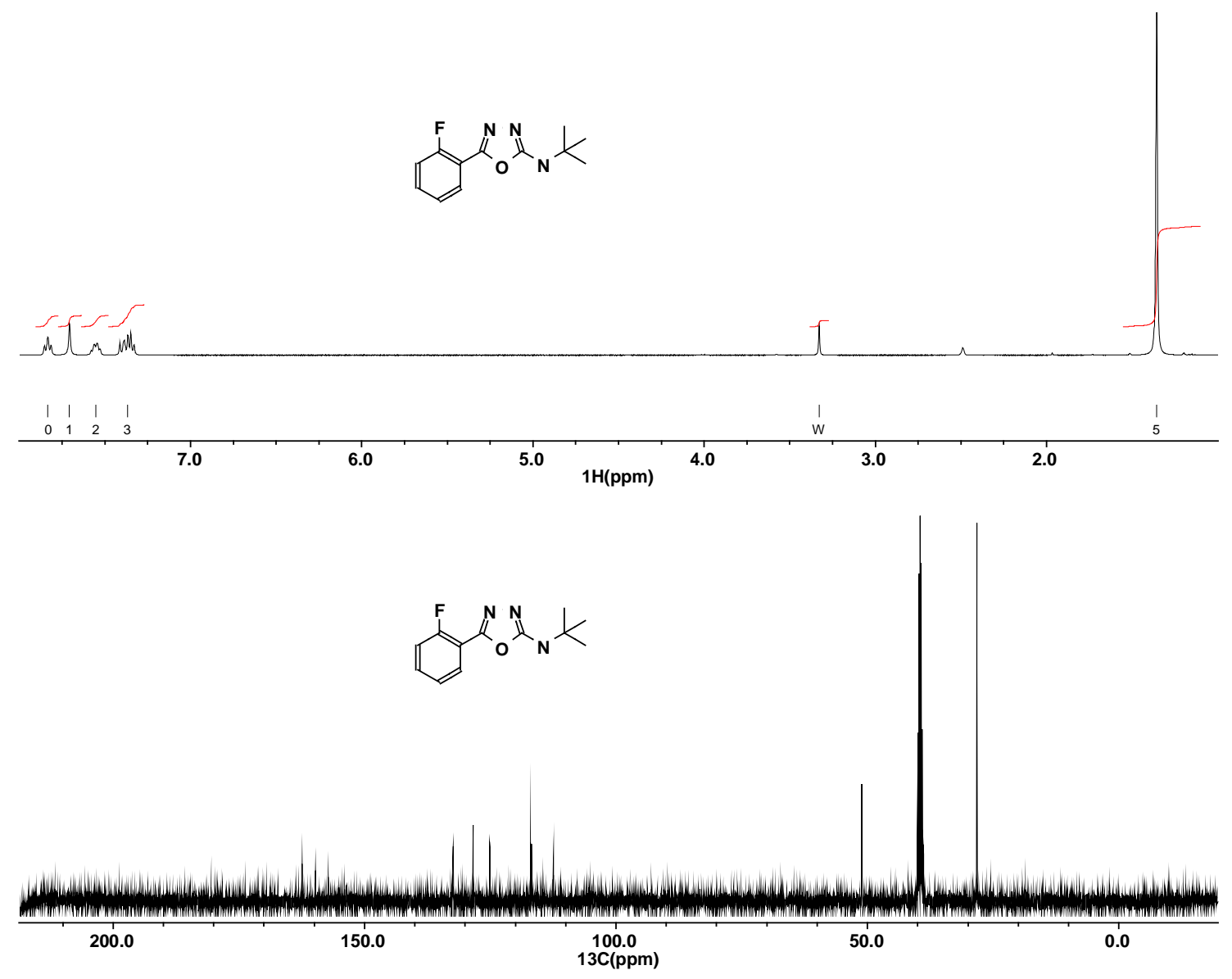


N-tert-Butyl-(5-(2-methylphenyl)-[1,3,4] oxadiazol-2-yl)-amine·HCl (16):

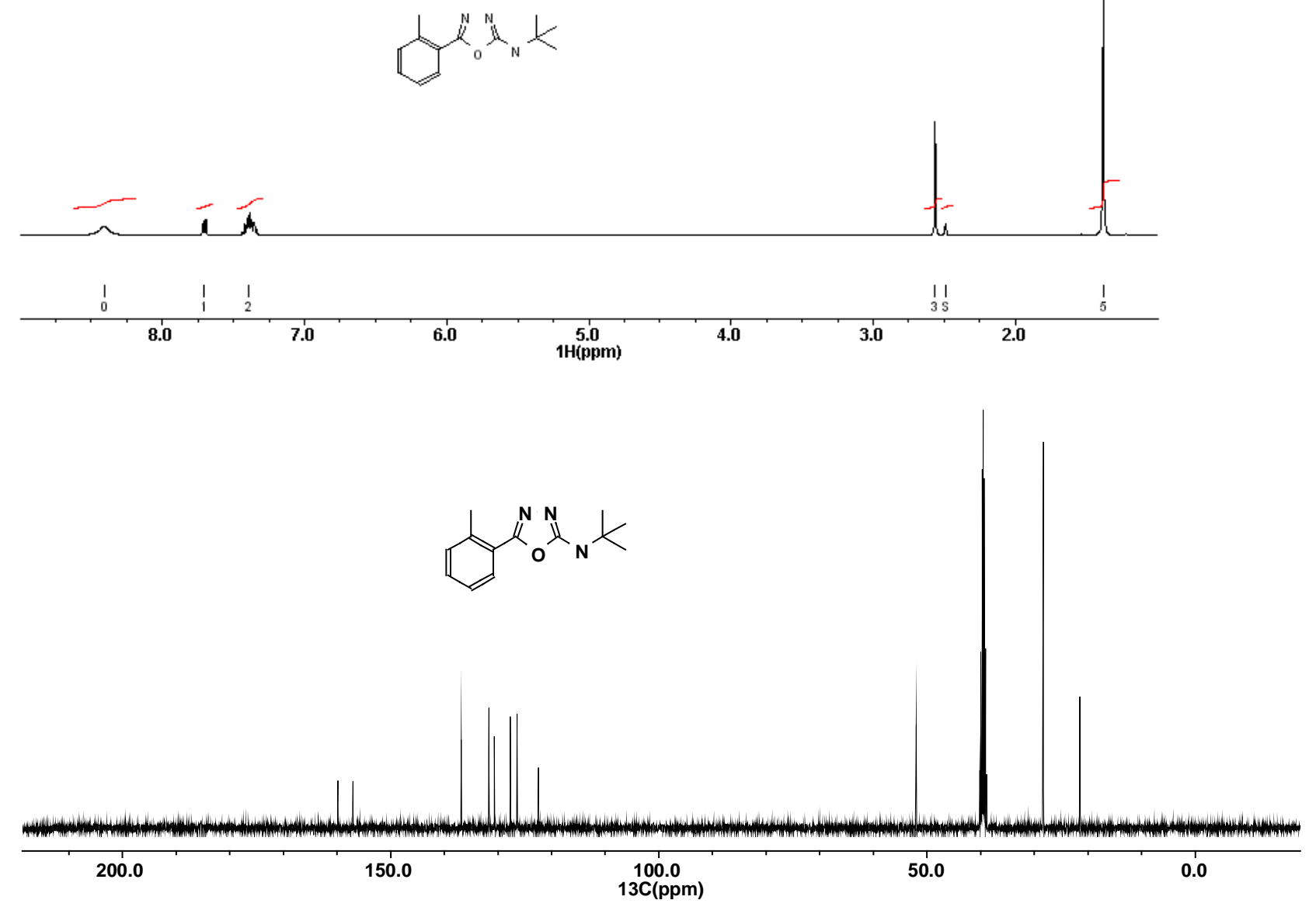


N-tert-Butyl-(5-(2-methoxy-phenyl)-[1,3,4]oxadiazol-2-yl)-amine-HCl (17):<smiles>COc1ccccc1C(=N)OC(=N)NC(C)(C)C</smiles>
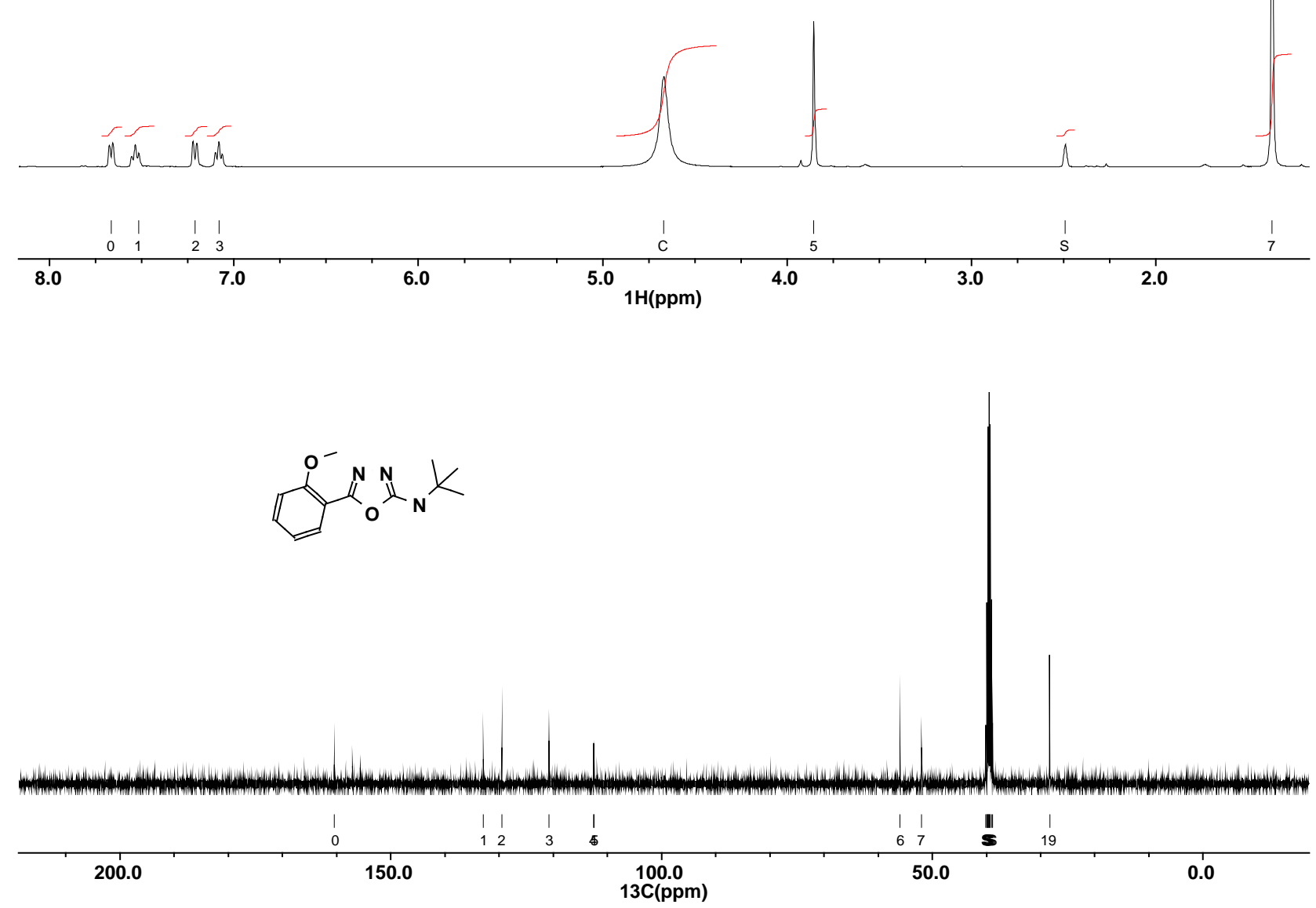
N-tert-Butyl-(5-(4-dimethylamino-phenyl)-[1,3,4] oxadiazol-2-yl)-amine (18):

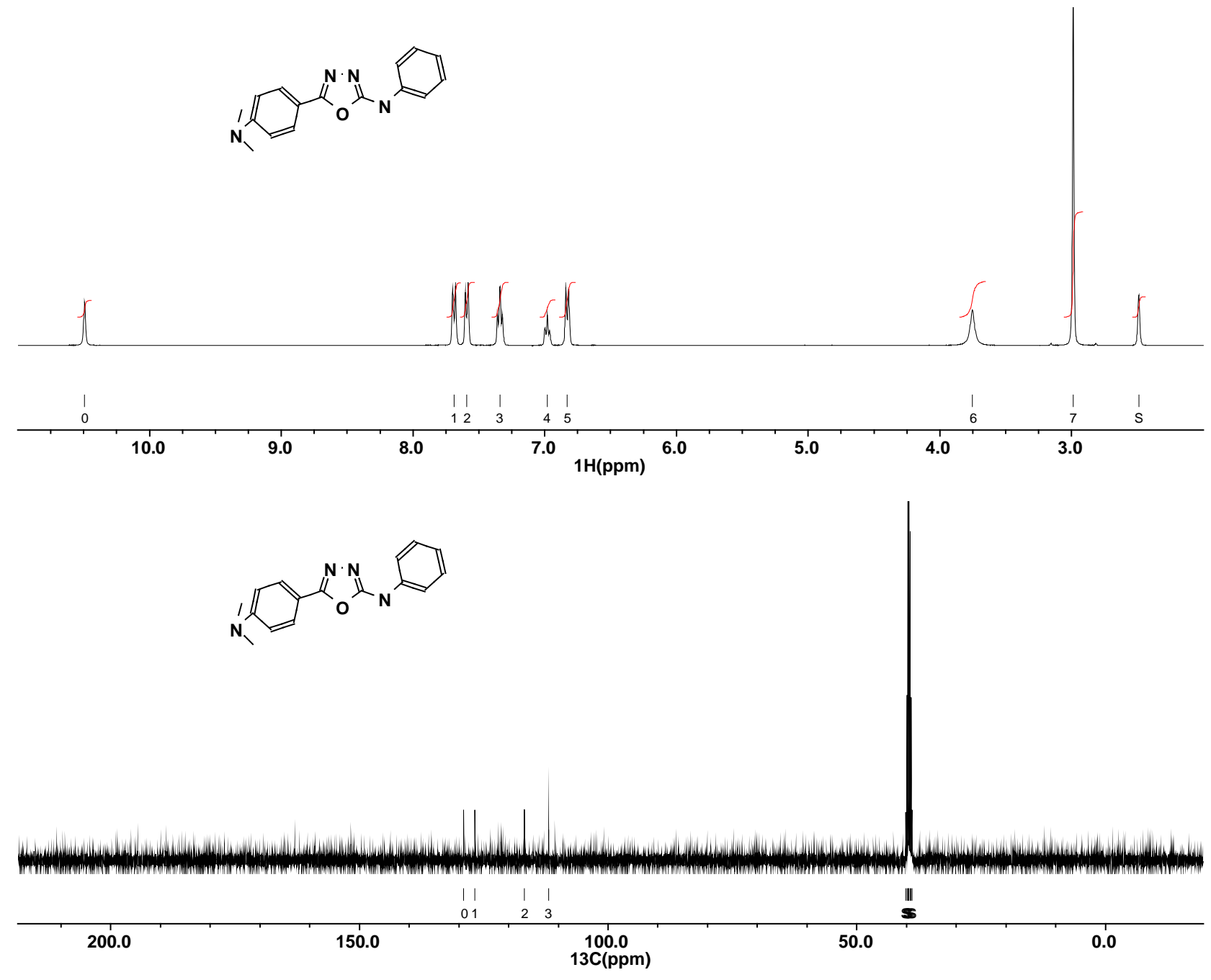


N-Phenyl-(5-(2-methoxy-phenyl)-[1,3,4]oxadiazol-2-yl)-amine·HCl (19):

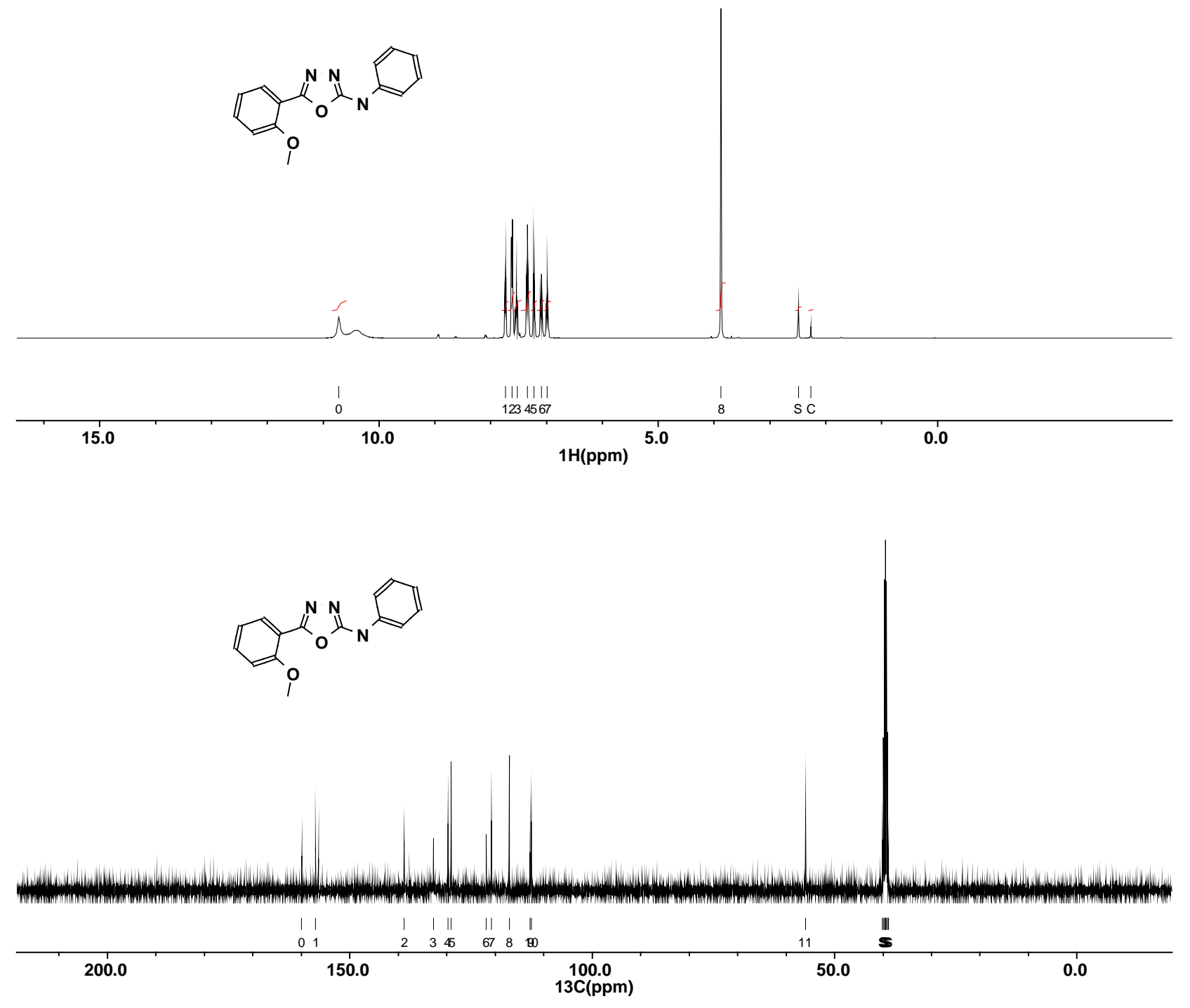


N-Phenyl acetic acid semicarbazide:

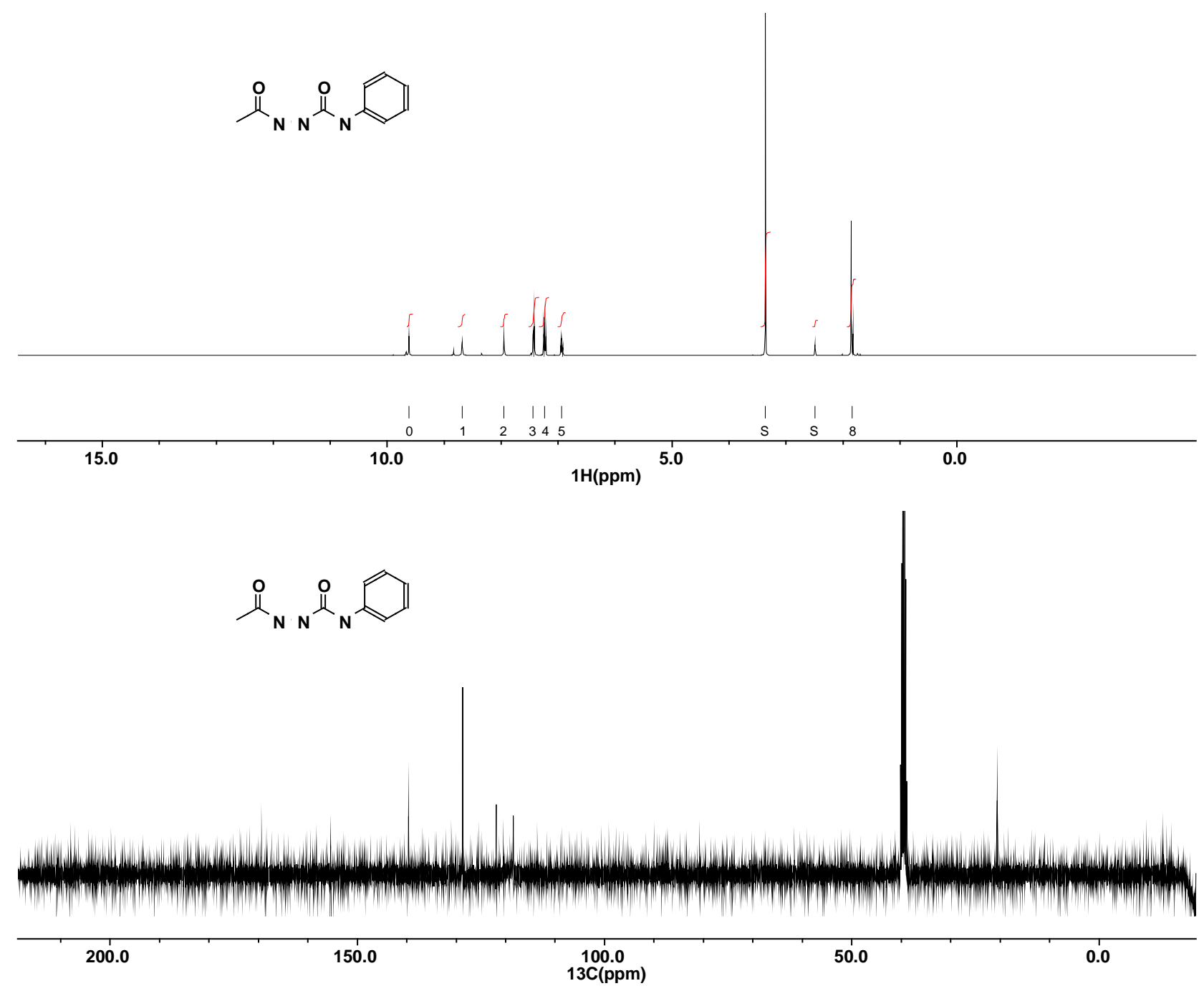


N-Phenyl acetic acid thiosemicarbazide:

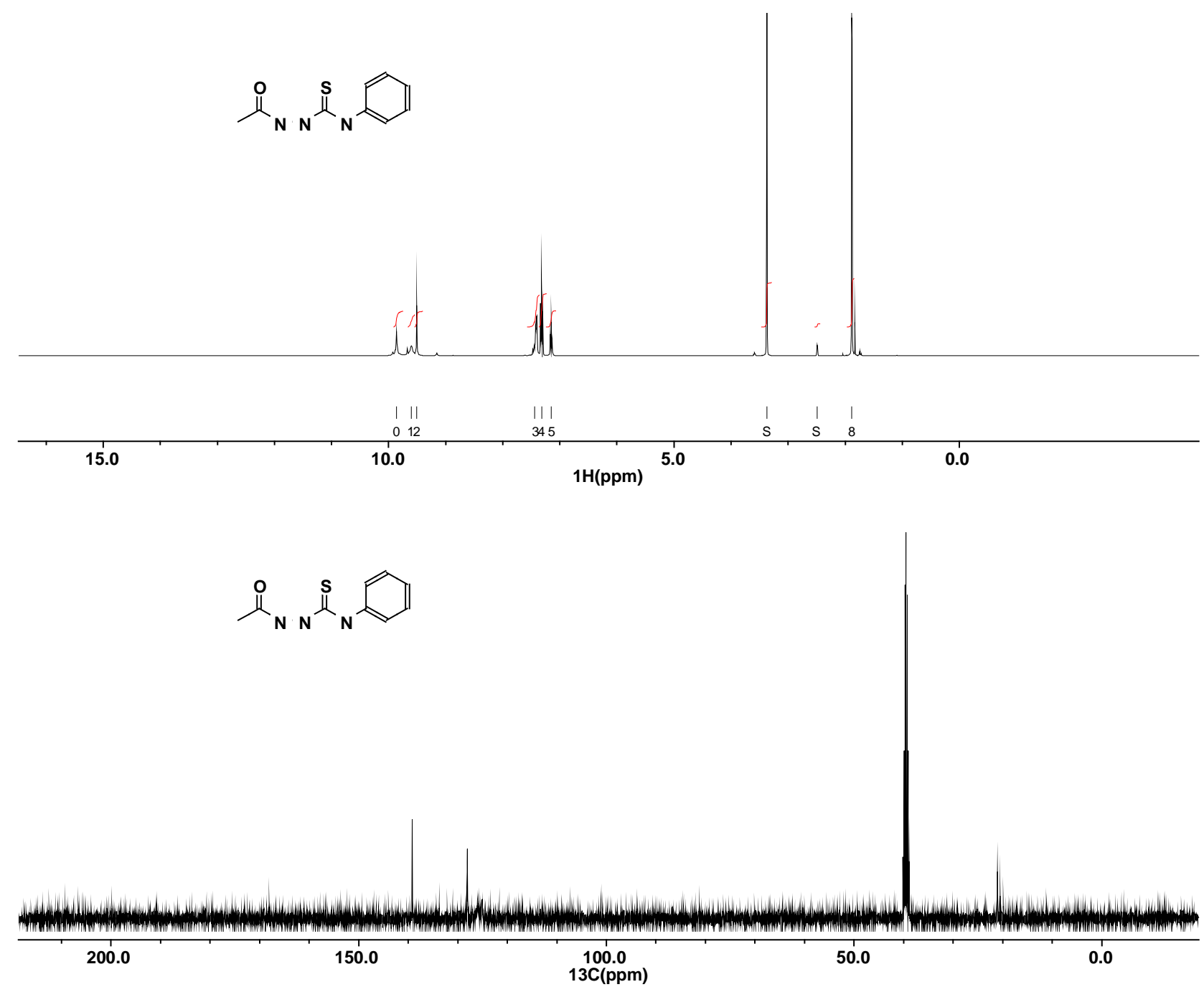


N-Phenyl-4-dimethylamino-benzoic acid semicarbazide:

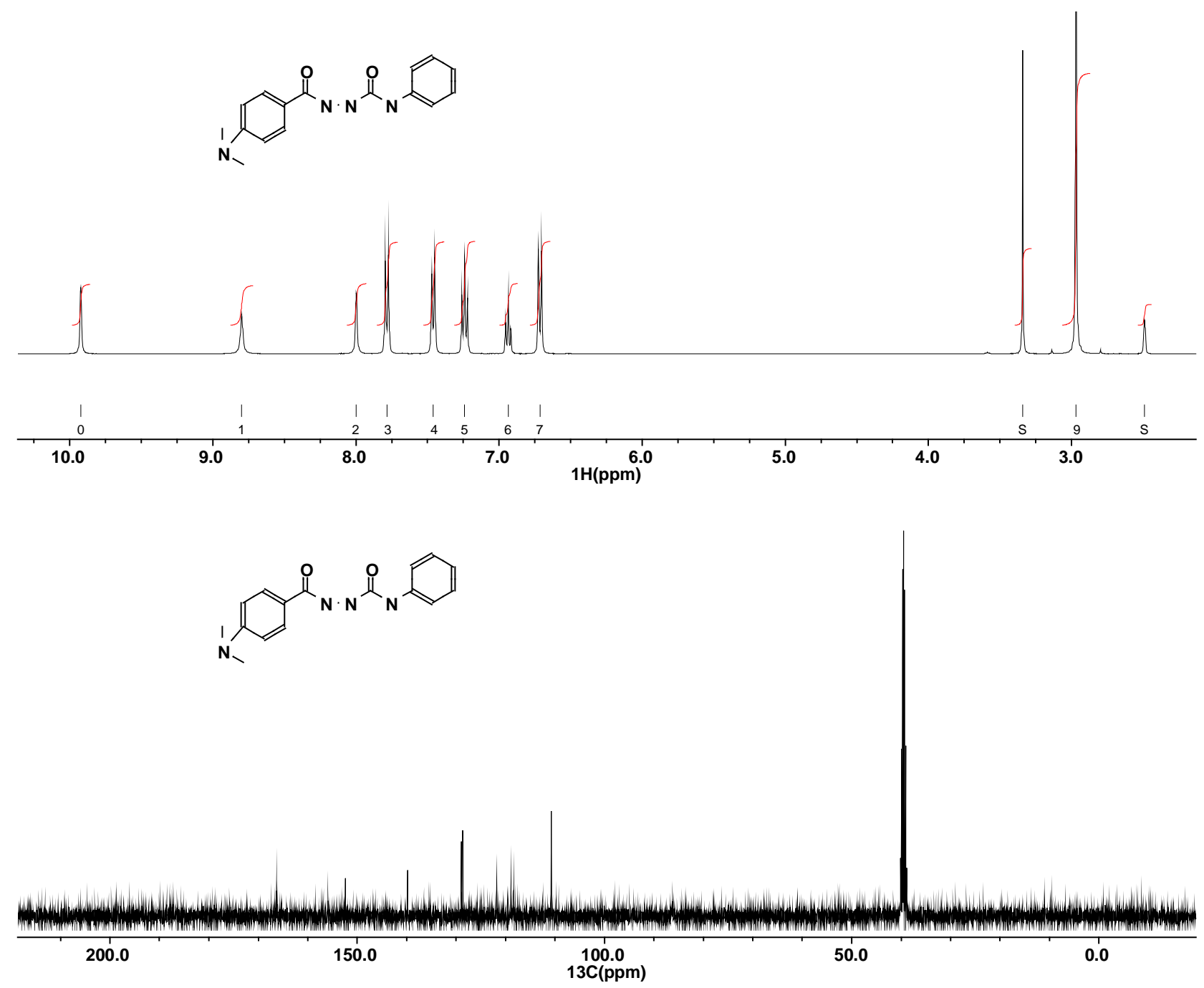


N-Phenyl-4-dimethylamino-benzoic acid thio-semicarbazide:

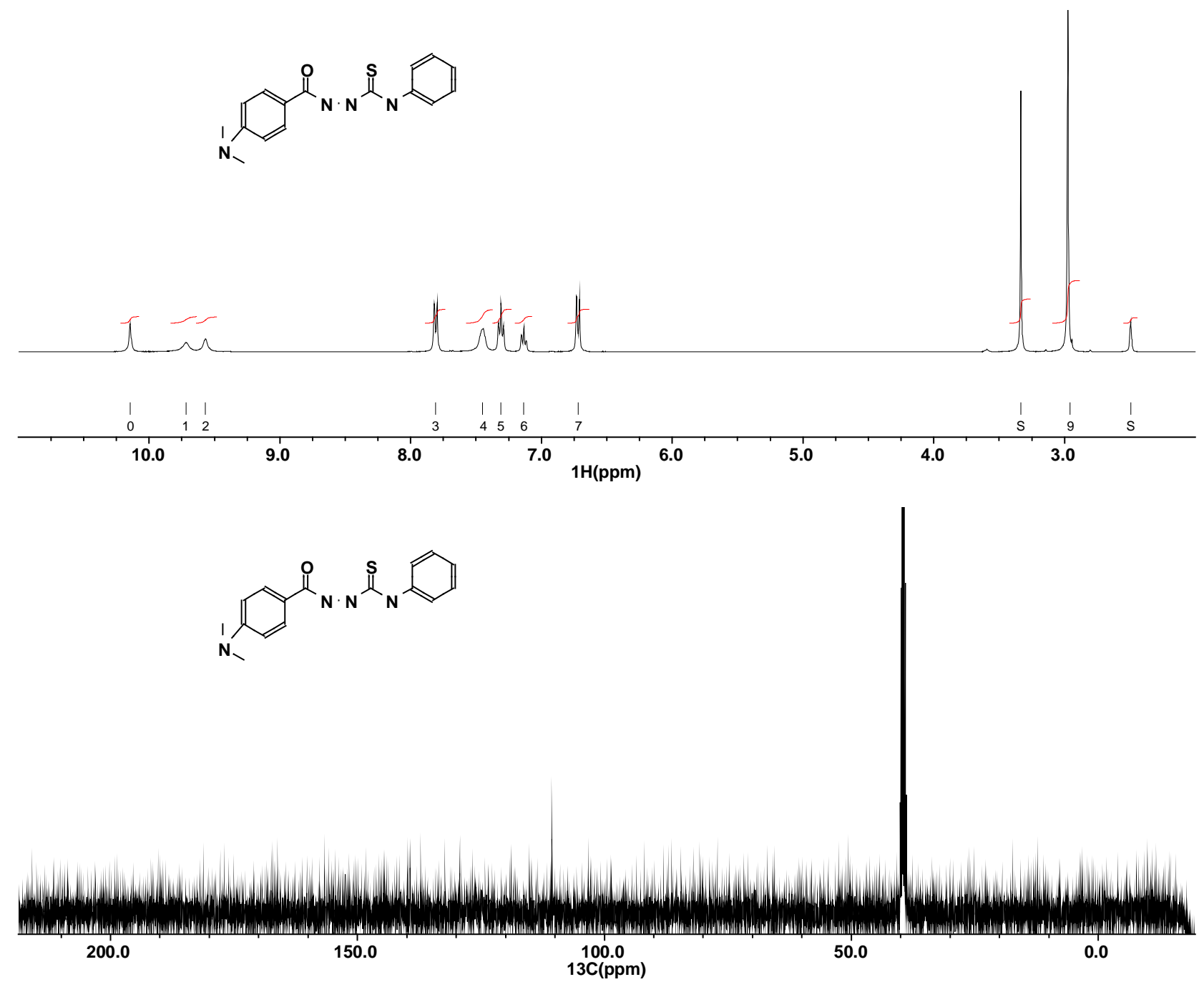

